\title{
Effect of Structure in Benzaldehyde Oximes on the Formation of Aldehydes and Nitriles under Photoinduced Electron Transfer Conditions
}

H. J. Peter de Lijser*, Susan Hsu, Bernadette V. Marquez, Adriana Park, Nawaporn Sanguantrakun, and Jody R. Sawyer

Department of Chemistry \& Biochemistry, California State University Fullerton, Fullerton, CA 92834-6866.

E-mail:pdelijser@fullerton.edu

\section{Supporting Information}

Plots of oxidation potential against polar and radical substituent constants

pp S2-S5

Hammett plots of relative quenching rates against polar and radical substituent constants

pp S6-S9

Hammett plots of relative oxime conversion against polar and radical substituent constants

Hammett plots of relative aldehyde yield against polar and radical substituent constants

pp S18-S25

Hammett plots of relative nitrile yield against polar and radical substituent constants

pp S26-S33

Plot of quenching rate $(\mathrm{kq})$ against the nitrile-aldehyde ratio

p S34

Plot of ionization potential against the nitrile aldehyde ratio

p S35

Tables of Cartesian coordinates for isomers A, B, and C of Compounds

$\mathbf{2 , 3}$, and 4

pp S36-S44 


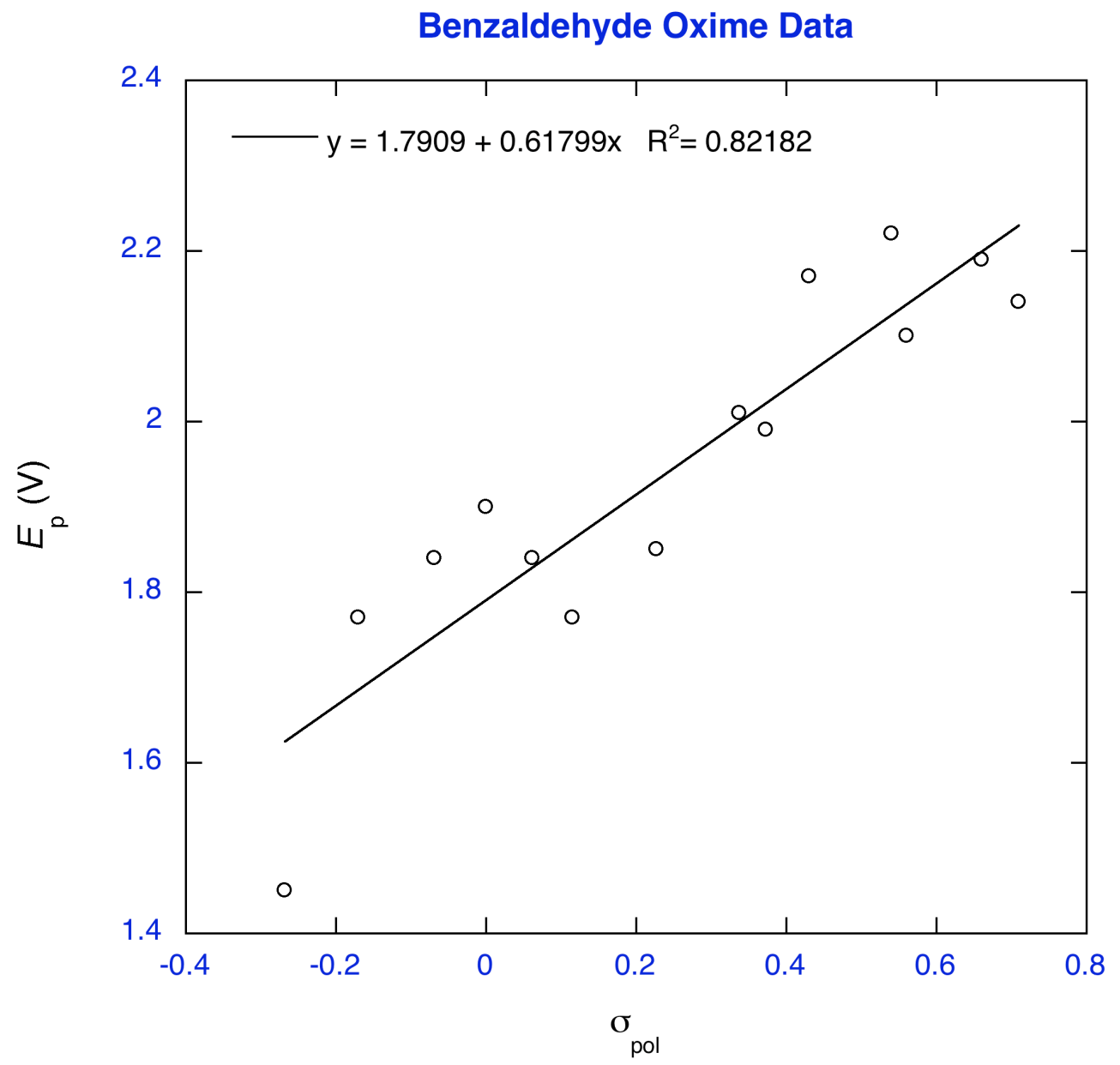

Figure S1. Plot of the measured oxidation potentials of meta- and para-substituted benzaldehyde oximes $\left(E_{\mathrm{p}}\right)$ versus the polar substituent constants $\sigma_{\mathrm{pol}}$. 


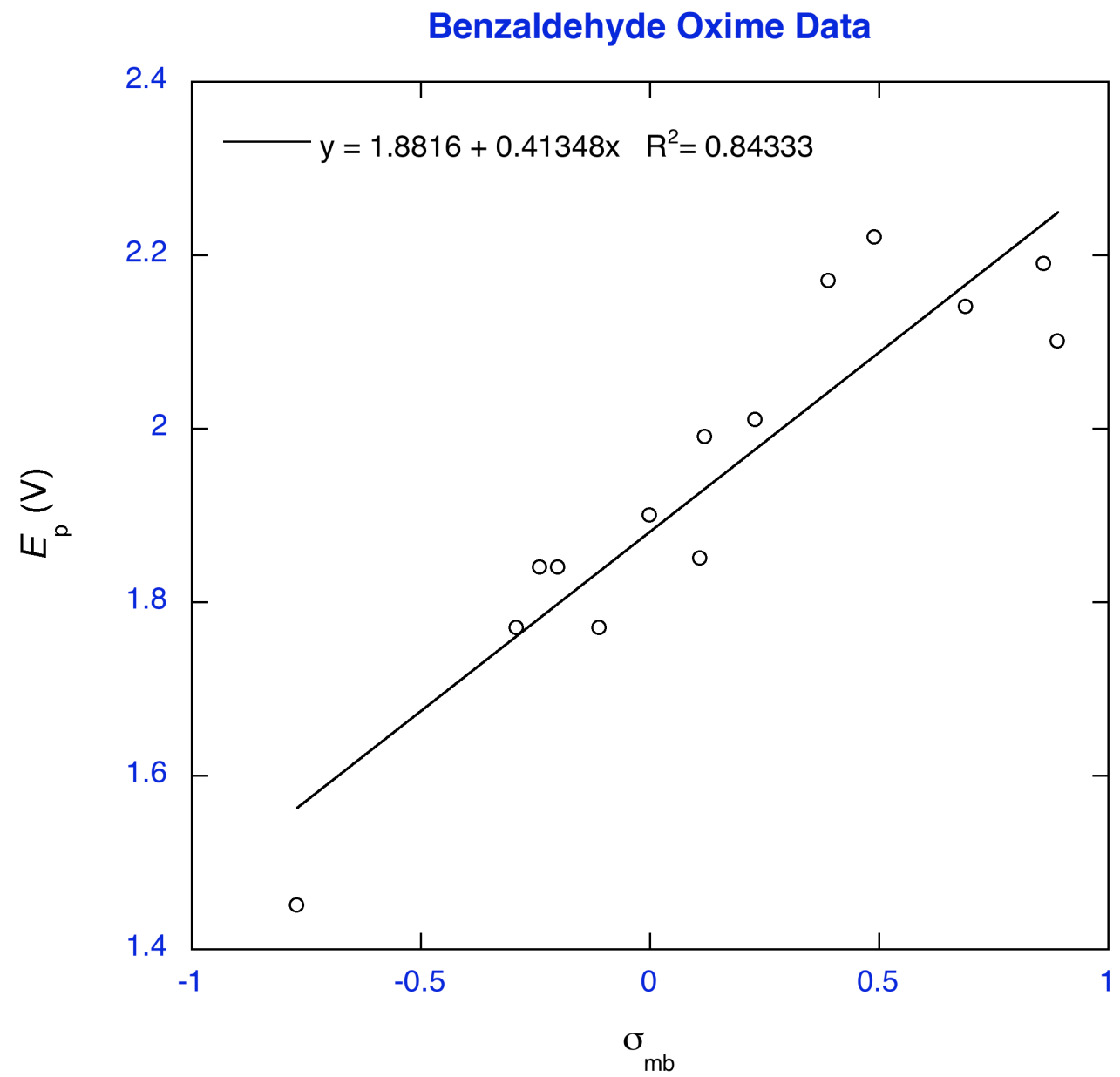

Figure S2. Plot of the measured oxidation potentials of meta- and para-substituted benzaldehyde oximes $\left(E_{\mathrm{p}}\right)$ versus the polar substituent constants $\sigma_{\mathrm{mb}}$. 


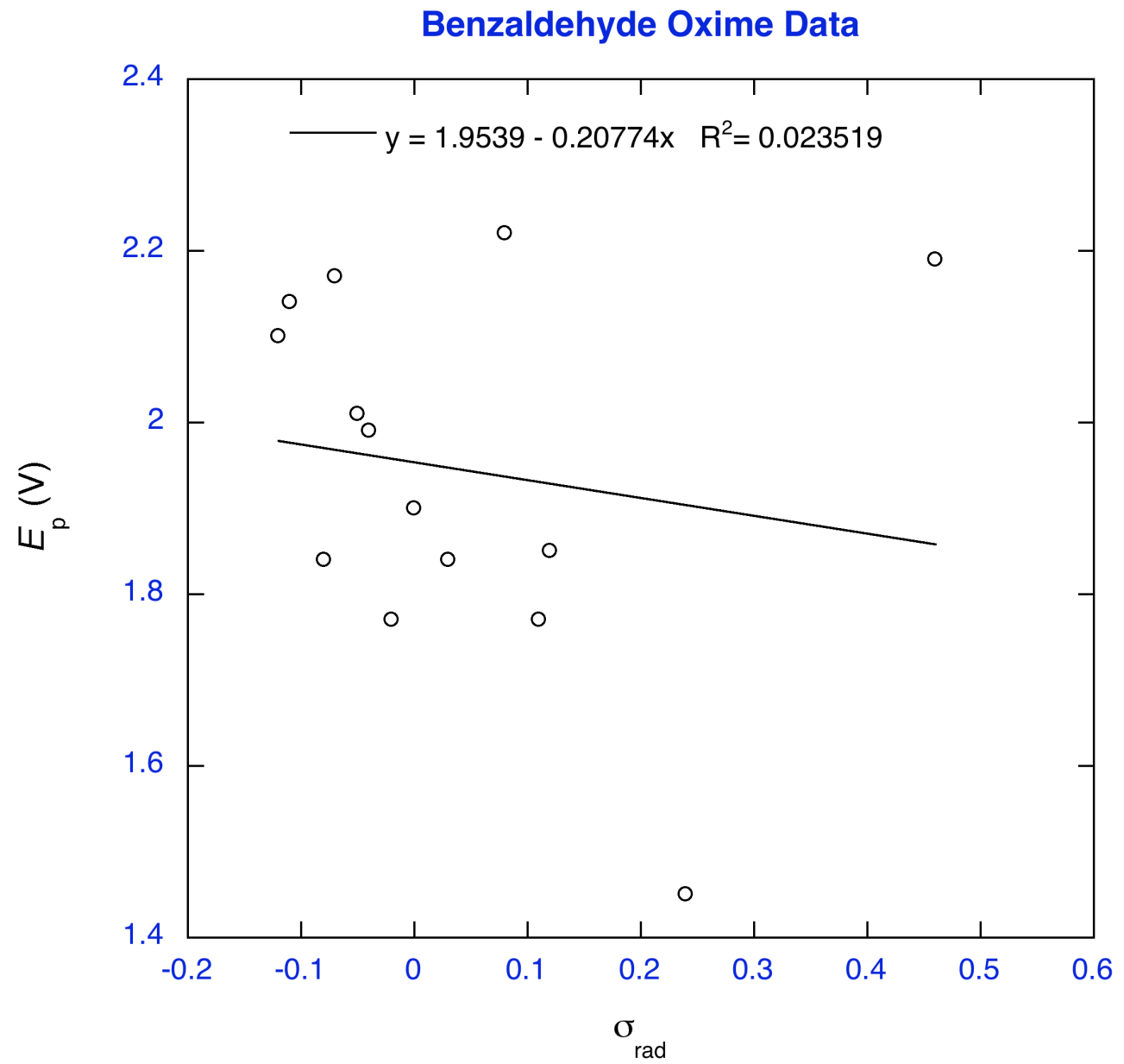

Figure S3. Plot of the measured oxidation potentials of meta- and para-substituted benzaldehyde oximes $\left(E_{\mathrm{p}}\right)$ versus the radical substituent constants $\sigma_{\text {rad. }}$. 


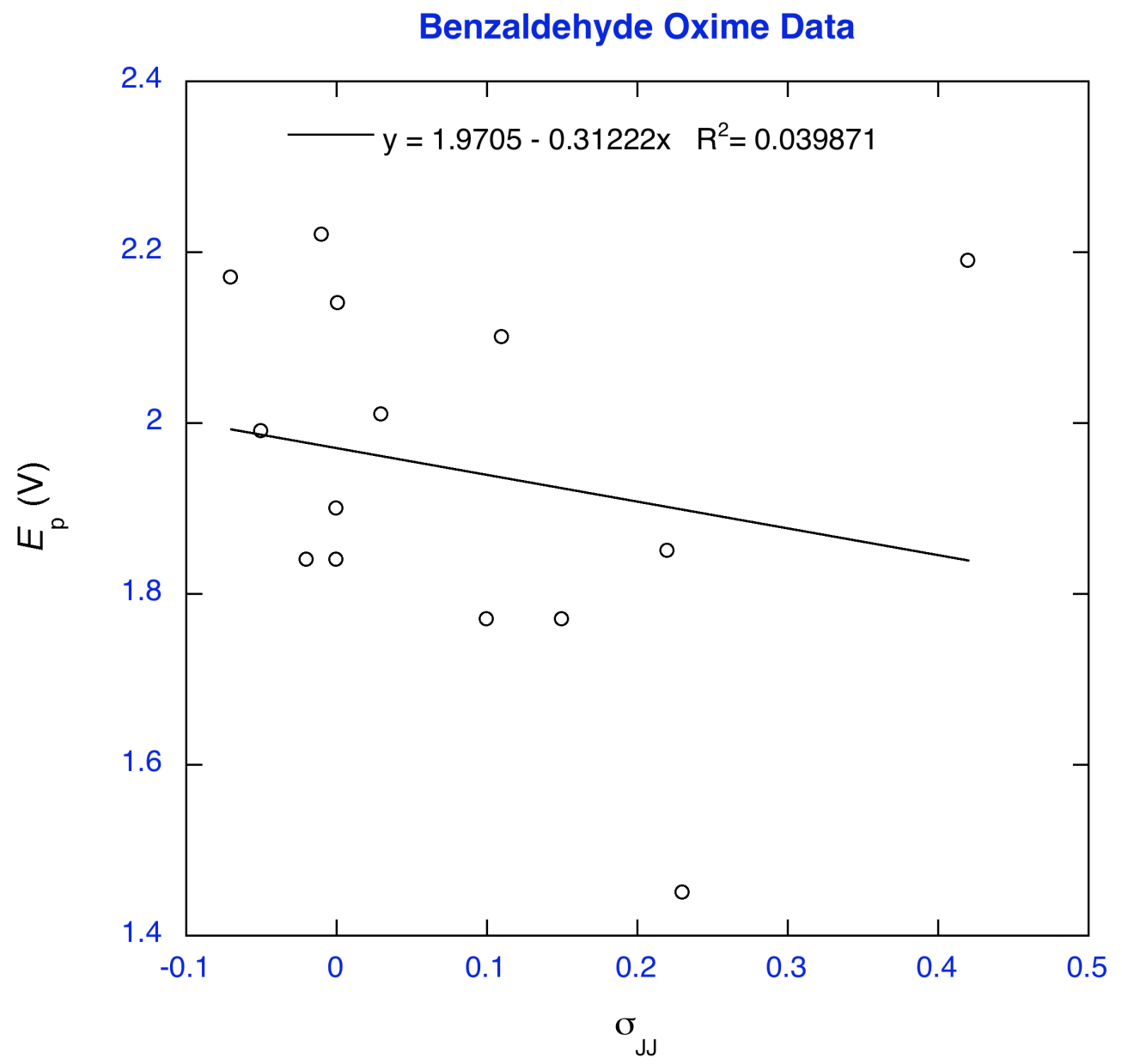

Figure S4. Plot of the measured oxidation potentials of meta- and para-substituted benzaldehyde oximes $\left(E_{\mathrm{p}}\right)$ versus the polar substituent constants $\sigma_{\mathrm{JJ}}$. 


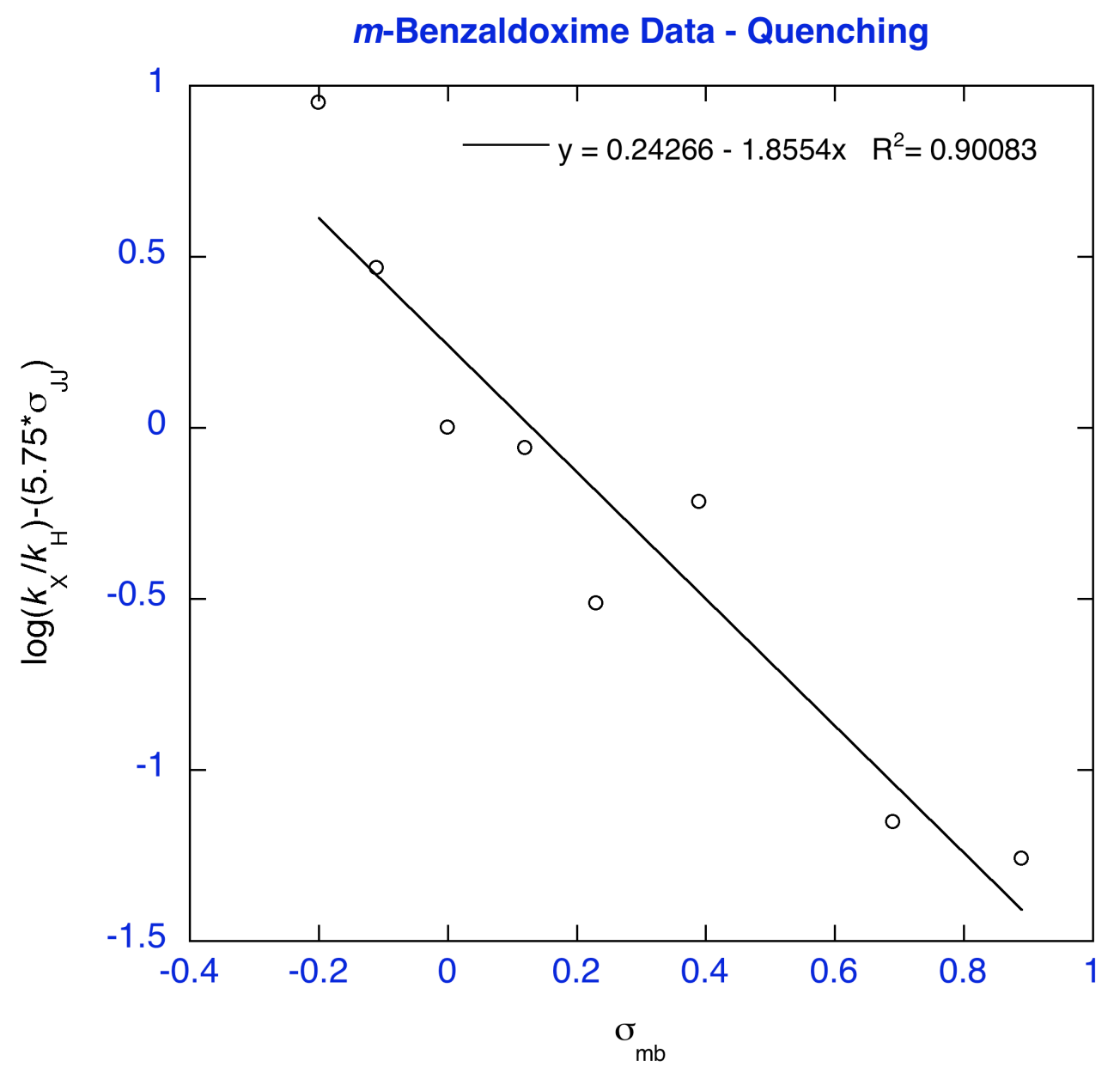

Figure S5. Dual-parameter semilogarithmic plot of the relative quenching rates of ${ }^{3} \mathrm{CA}\left(k_{\mathrm{X}} / k_{\mathrm{H}}\right)$ by meta-substituted benzaldehyde oximes versus radical $\left(\sigma_{\mathrm{JJ}}\right)$ and polar $\left(\sigma_{\mathrm{mb}}\right)$ substituent constants. 


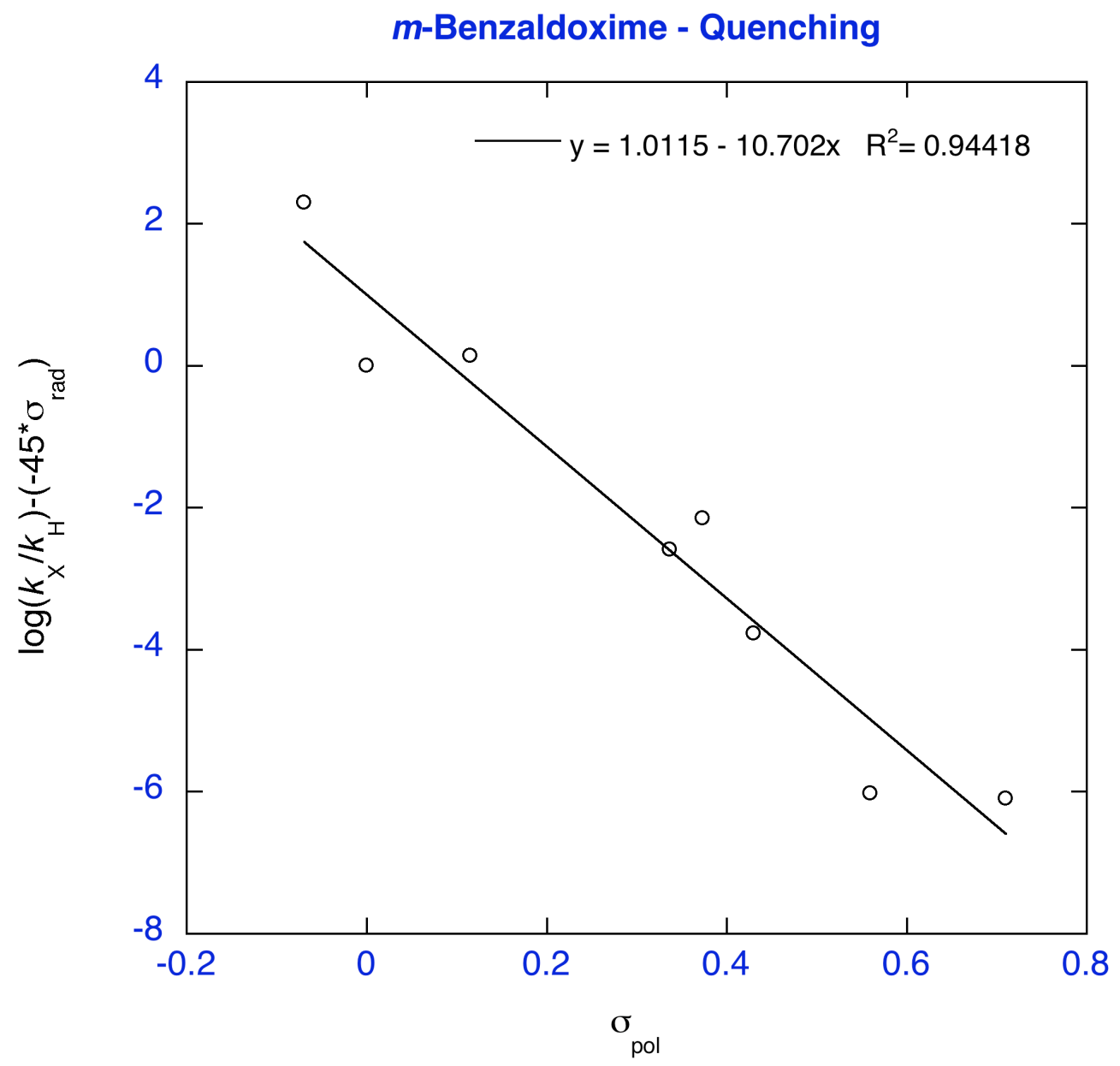

Figure S6. Dual-parameter semilogarithmic plot of the relative quenching rates of ${ }^{3} \mathrm{CA}\left(k_{\mathrm{X}} / k_{\mathrm{H}}\right)$ by meta-substituted benzaldehyde oximes versus radical $\left(\sigma_{\text {rad }}\right)$ and polar $\left(\sigma_{\text {pol }}\right)$ substituent constants. 


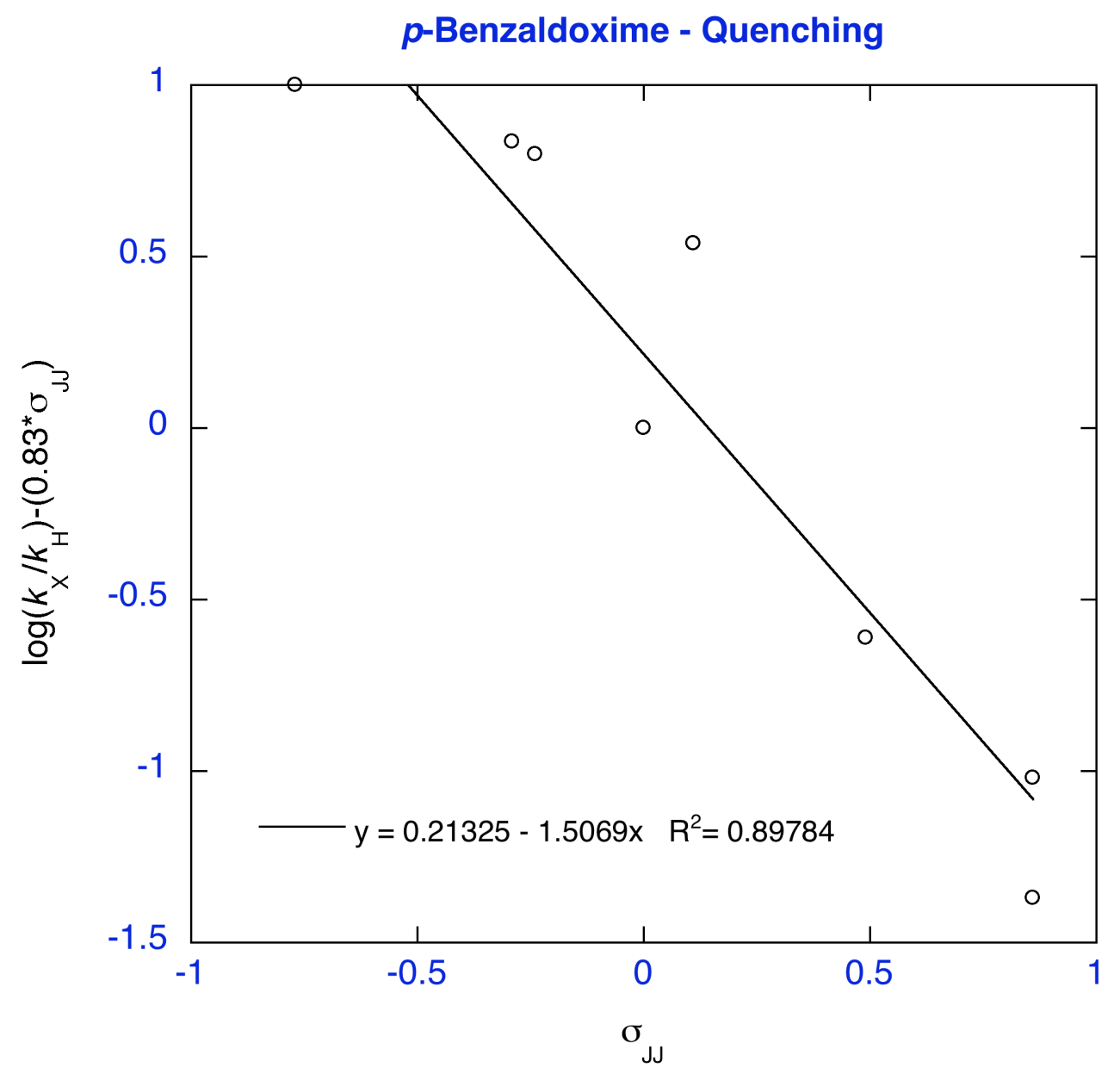

Figure S7. Dual-parameter semilogarithmic plot of the relative quenching rates of ${ }^{3} \mathrm{CA}\left(k_{\mathrm{X}} / k_{\mathrm{H}}\right)$ by para-substituted benzaldehyde oximes versus radical $\left(\sigma_{\mathrm{JJ}}\right)$ and polar $\left(\sigma_{\mathrm{mb}}\right)$ substituent constants. 


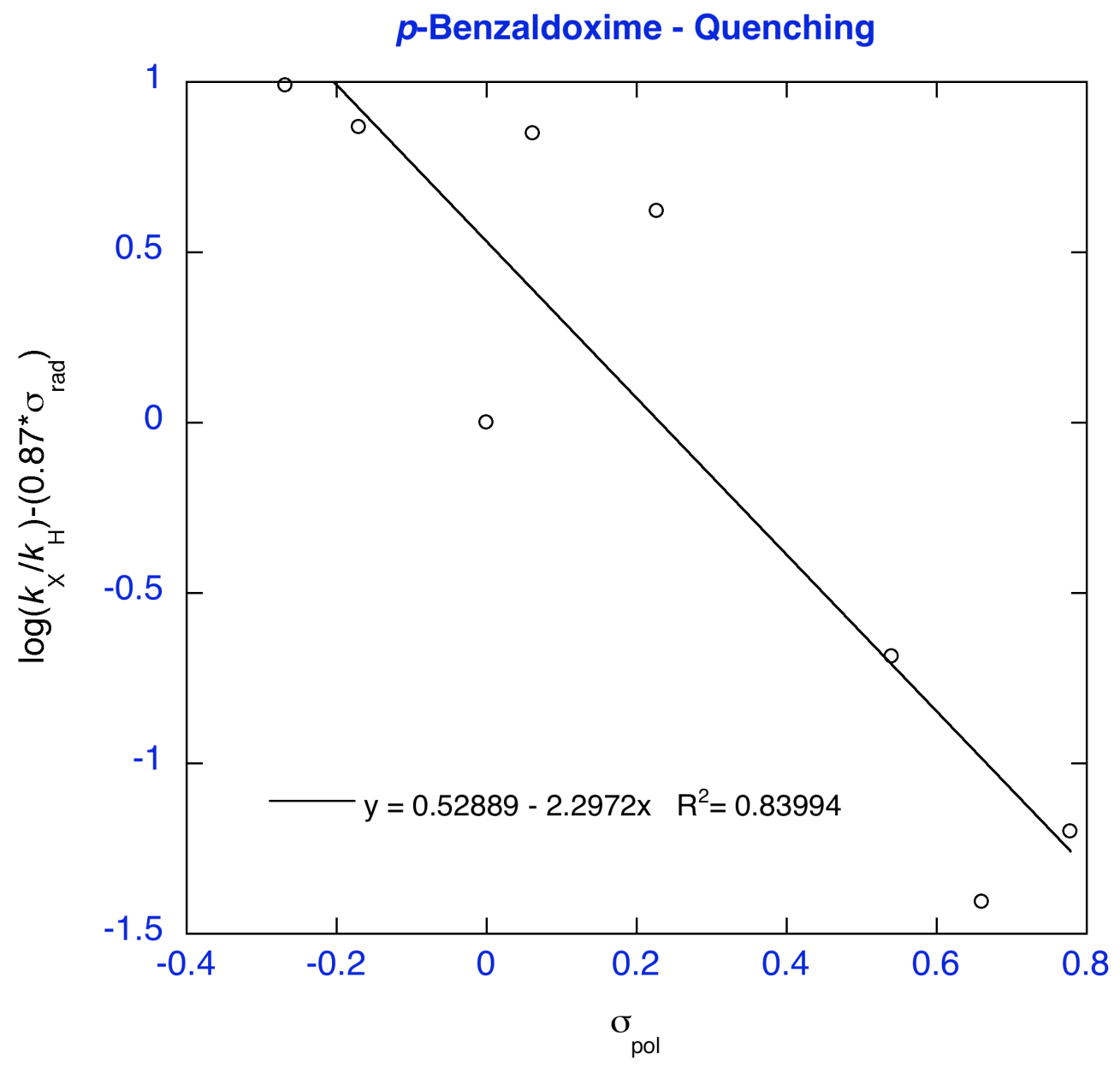

Figure S8. Dual-parameter semilogarithmic plot of the relative quenching rates of ${ }^{3} \mathrm{CA}\left(k_{\mathrm{X}} / k_{\mathrm{H}}\right)$ by para-substituted benzaldehyde oximes versus radical $\left(\sigma_{\text {rad }}\right)$ and polar $\left(\sigma_{\text {pol }}\right)$ substituent constants. 


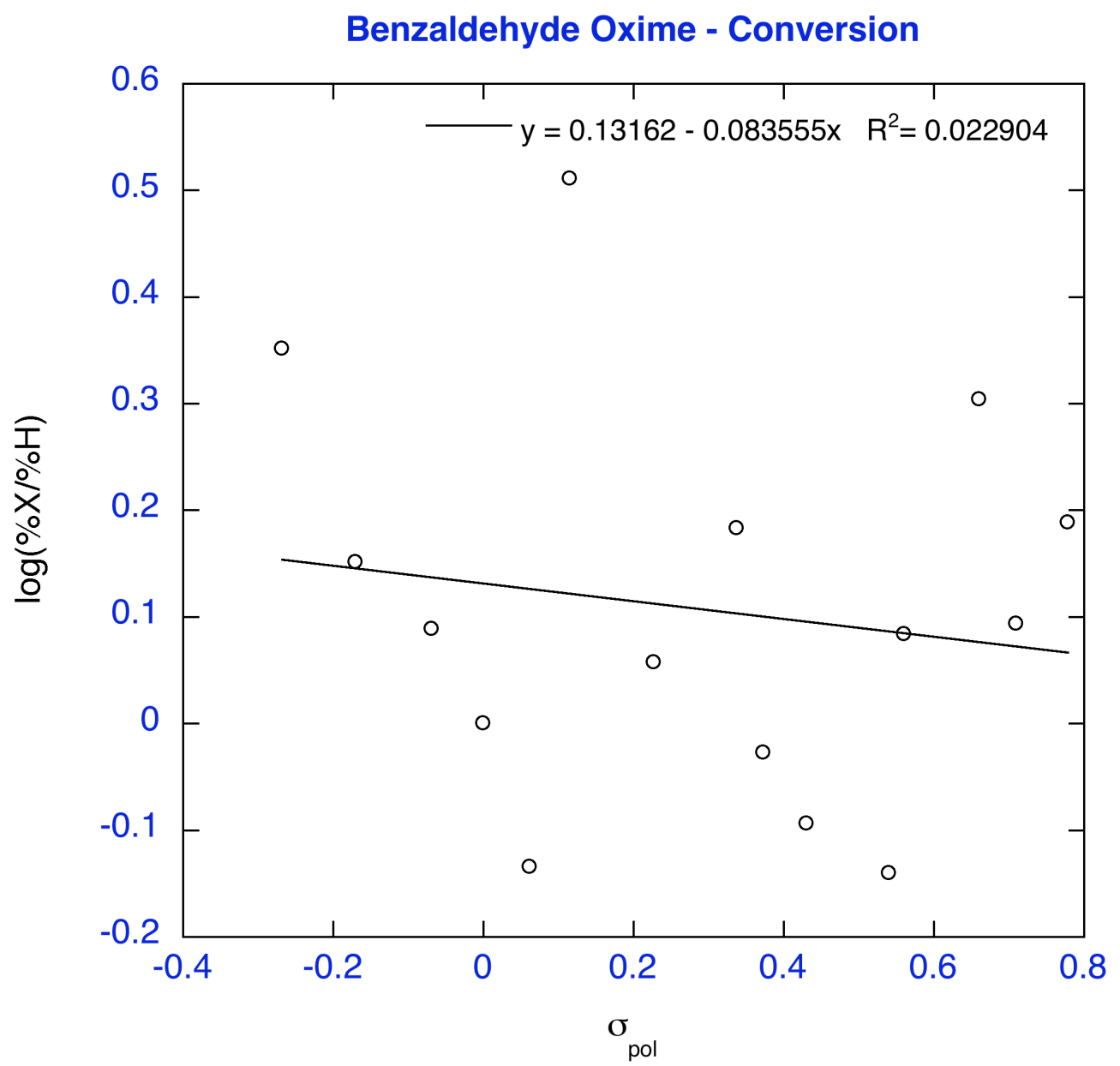

Figure S9. Semilogarithmic plot of the relative conversion of meta- and para-substituted benzaldehyde oximes $\left(\% \mathrm{x} / \%_{\mathrm{H}}\right)$ versus the polar substituent constant $\sigma_{\mathrm{pol}}$. 


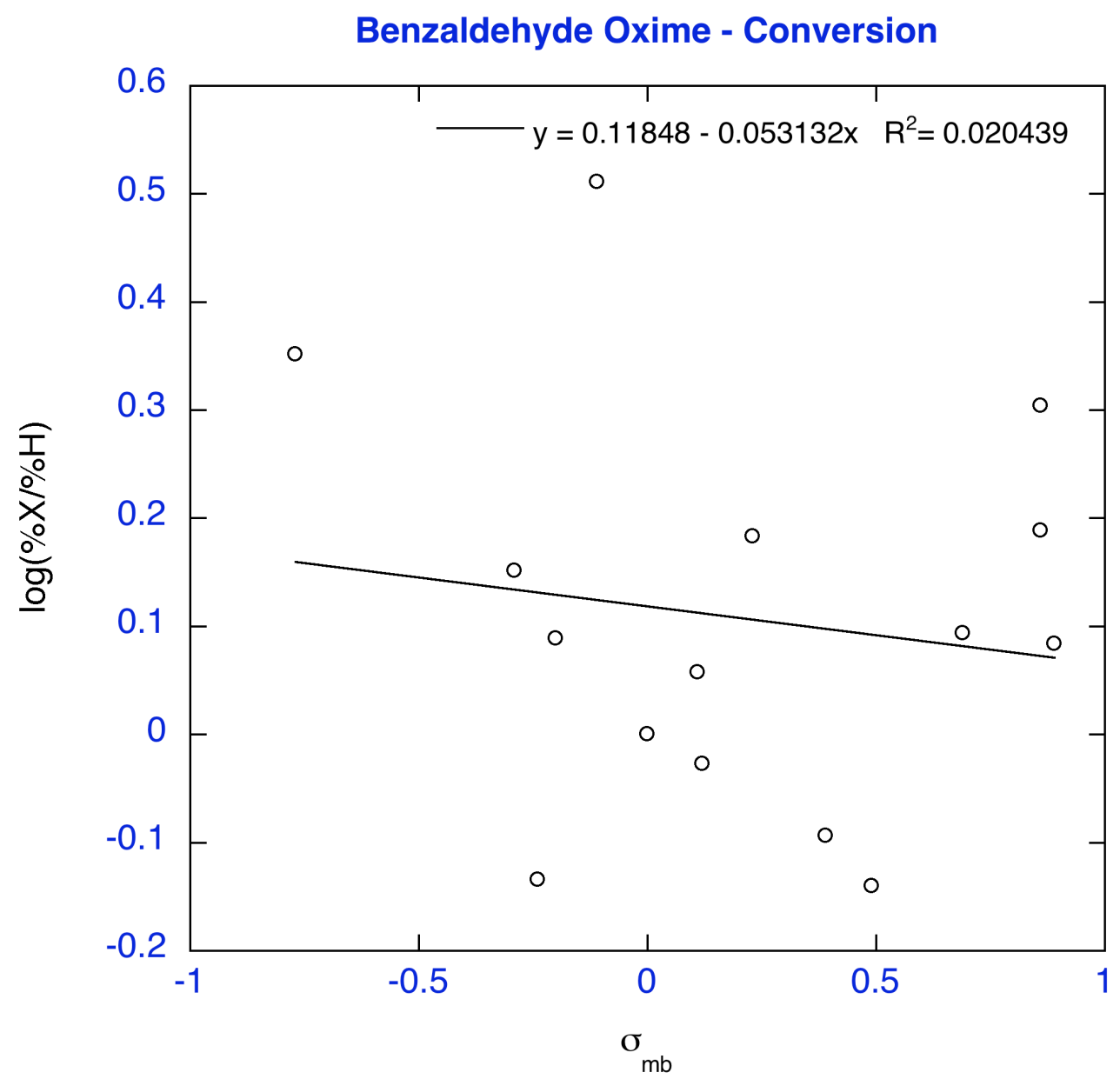

Figure S10. Semilogarithmic plot of the relative conversion of meta- and para-substituted benzaldehyde oximes $\left(\% \mathrm{x} / \%_{\mathrm{H}}\right)$ versus the polar substituent constant $\sigma_{\mathrm{mb}}$. 


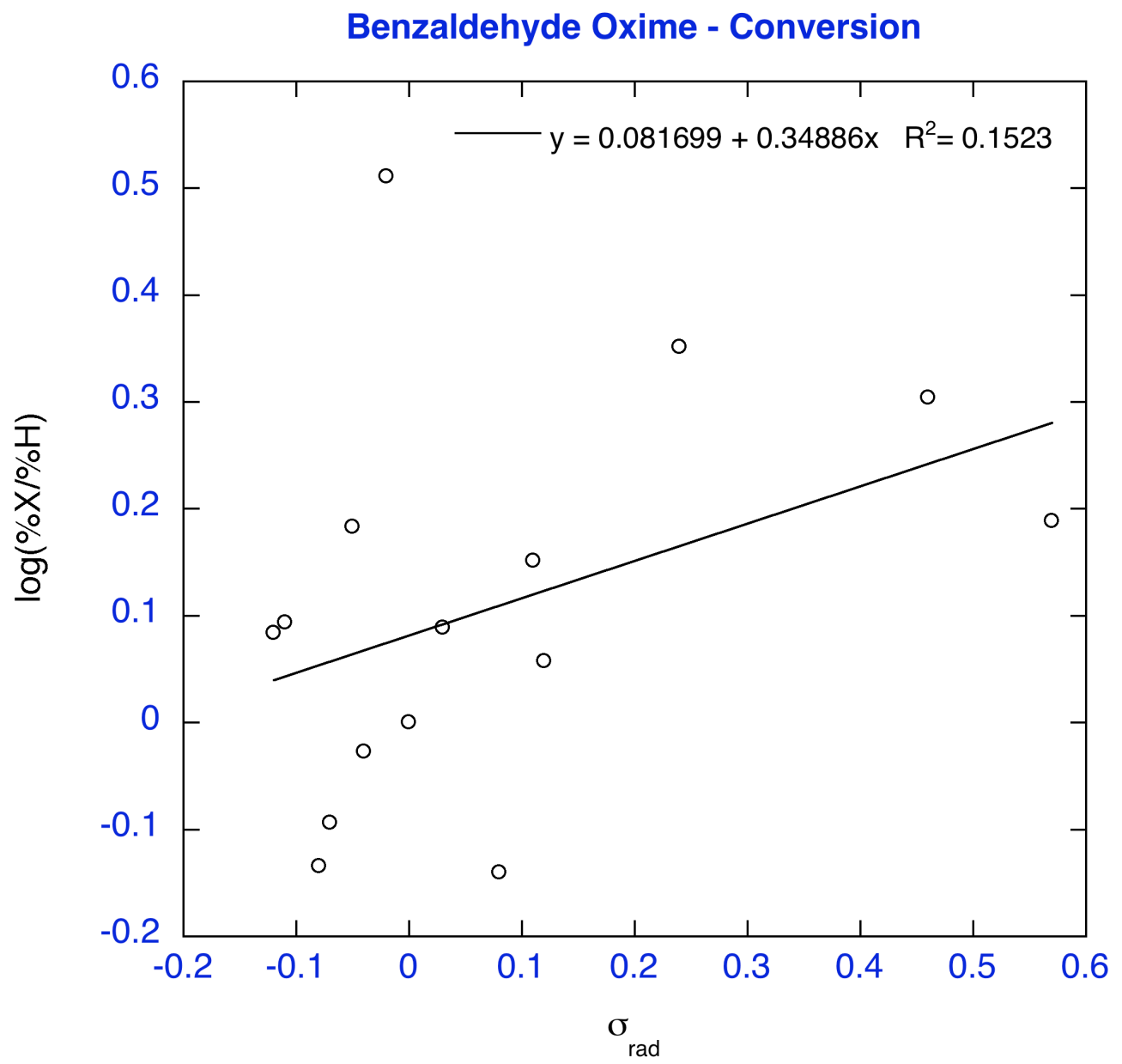

Figure S11. Semilogarithmic plot of the relative conversion of meta- and para-substituted benzaldehyde oximes $\left(\%_{\mathrm{X}} / \%_{\mathrm{H}}\right)$ versus the radical substituent constant $\sigma_{\text {rad. }}$. 


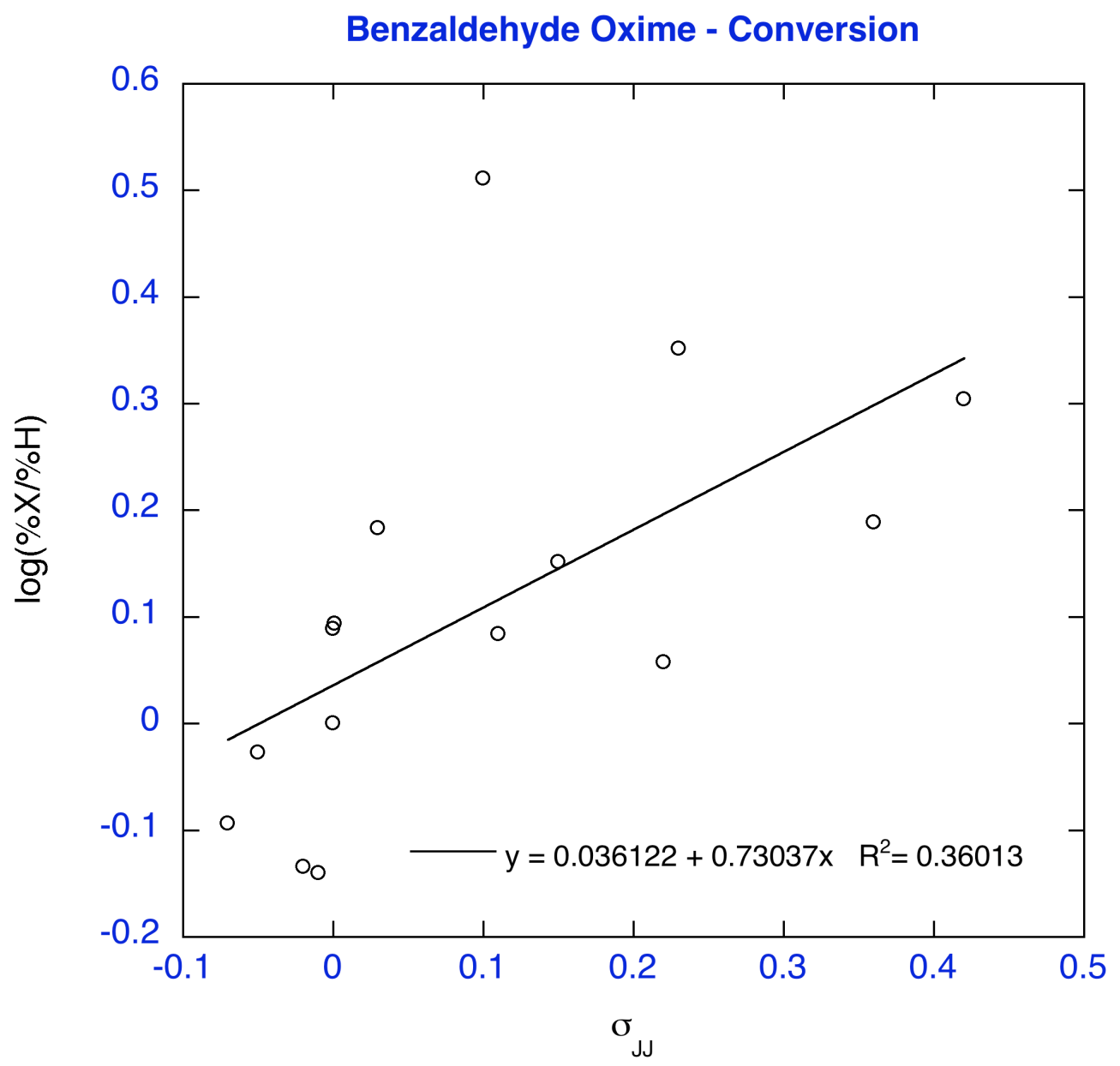

Figure S12. Semilogarithmic plot of the relative conversion of meta- and para-substituted benzaldehyde oximes $\left(\% \mathrm{X} / \%_{\mathrm{H}}\right)$ versus the radical substituent constant $\sigma_{\mathrm{JJ}}$. 


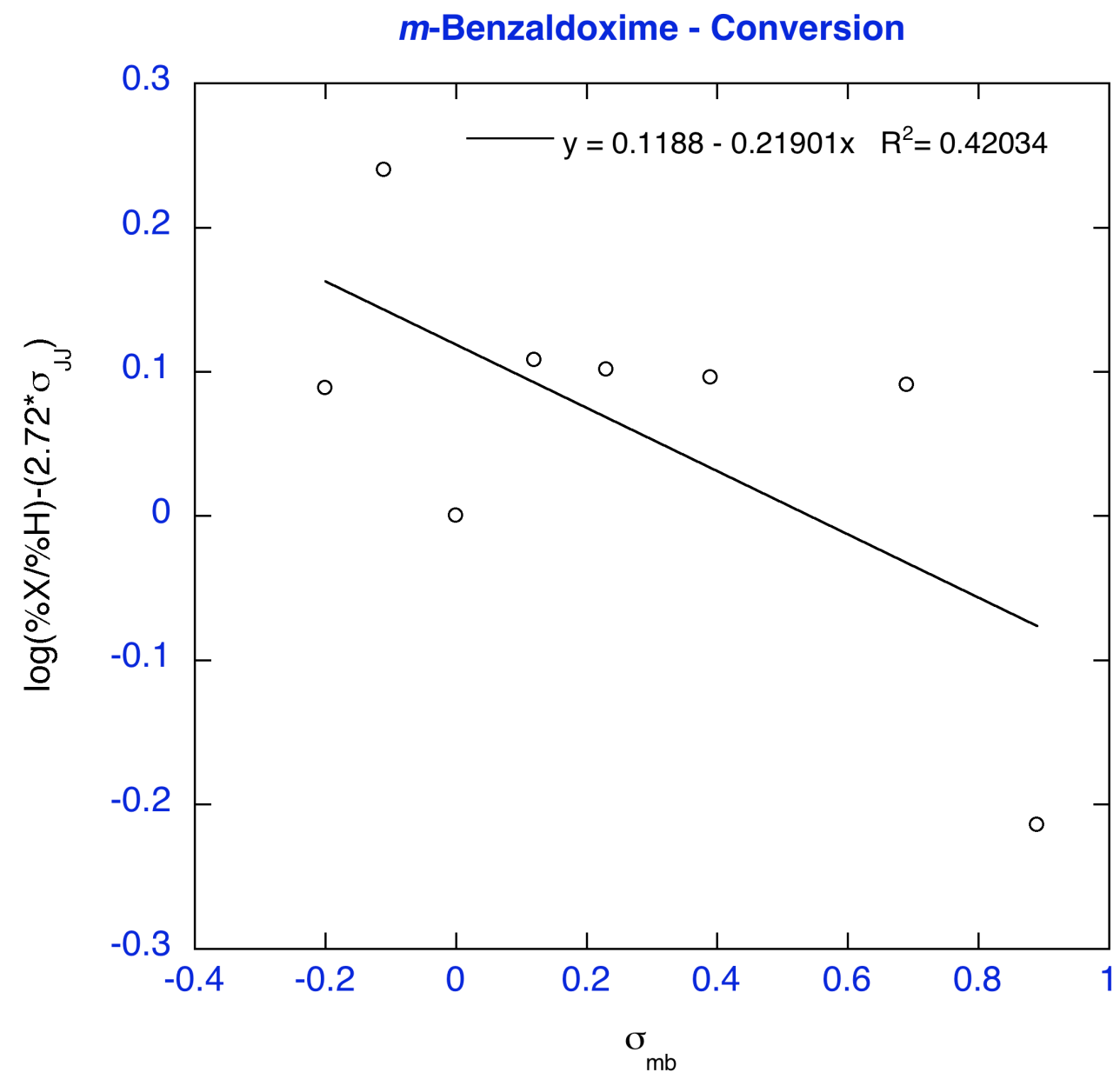

Figure S13. Dual-parameter semilogarithmic plot of the relative conversion of meta-substituted benzaldehyde oximes $\left(\% \mathrm{X} / \%_{\mathrm{H}}\right)$ versus radical $\left(\sigma_{\mathrm{JJ}}\right)$ and polar $\left(\sigma_{\mathrm{mb}}\right)$ substituent constants. 


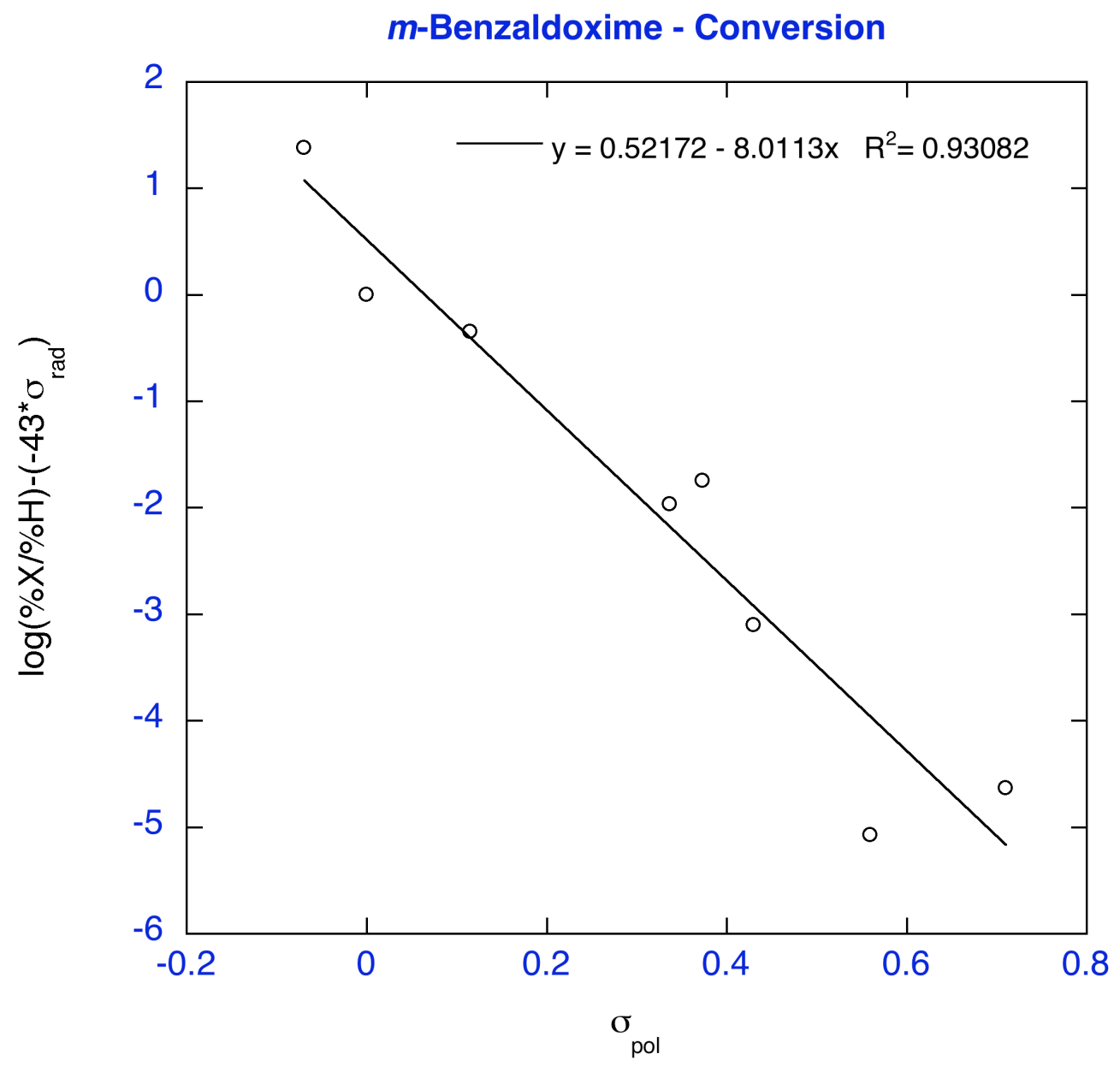

Figure S14. Dual-parameter semilogarithmic plot of the relative conversion of meta-substituted benzaldehyde oximes $\left(\%_{\mathrm{X}} / \%_{\mathrm{H}}\right)$ versus radical $\left(\sigma_{\mathrm{rad}}\right)$ and polar $\left(\sigma_{\mathrm{pol}}\right)$ substituent constants. 


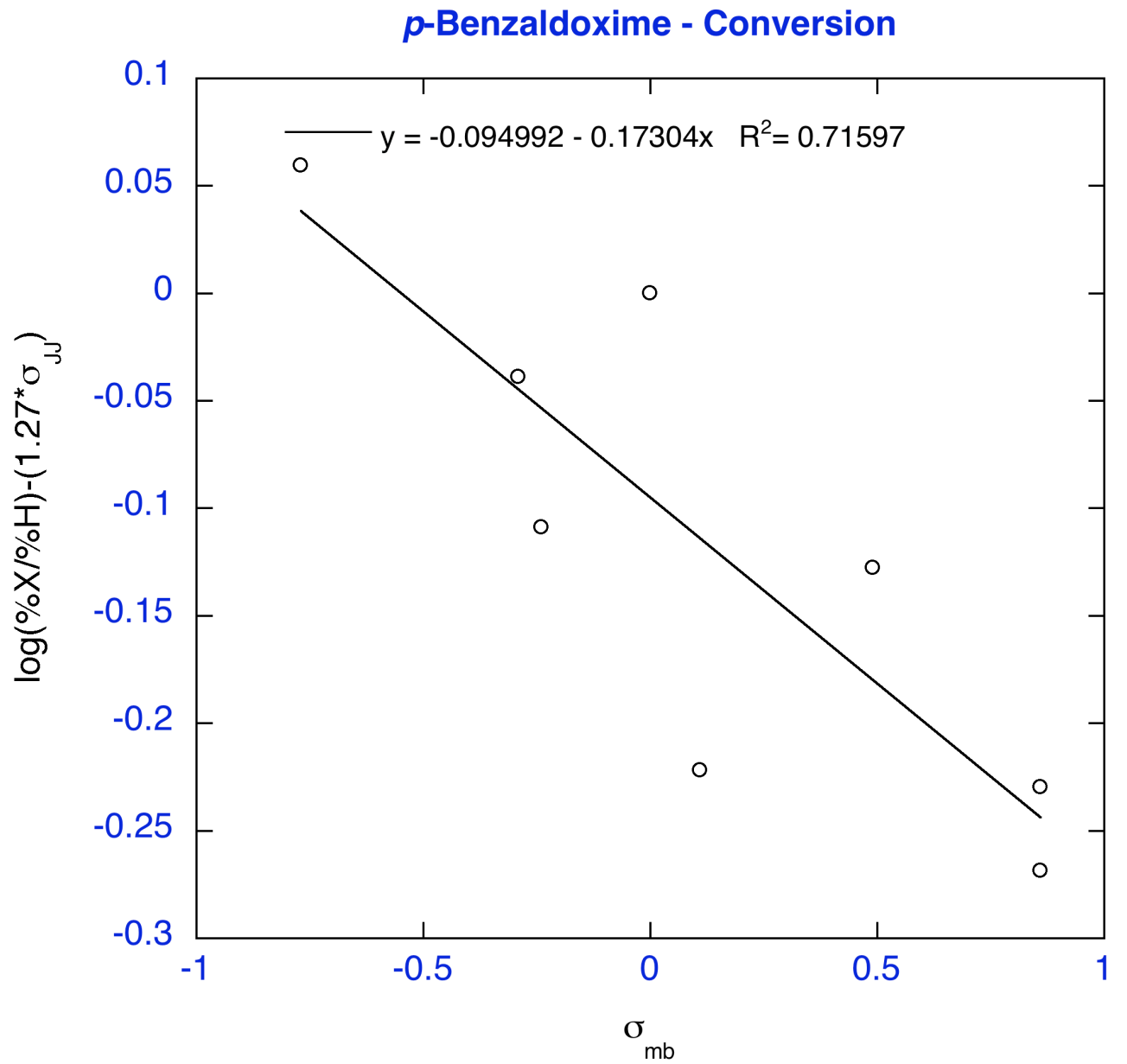

Figure S15. Dual-parameter semilogarithmic plot of the relative conversion of para-substituted benzaldehyde oximes $\left(\% \mathrm{X} / \%_{\mathrm{H}}\right)$ versus radical $\left(\sigma_{\mathrm{JJ}}\right)$ and polar $\left(\sigma_{\mathrm{mb}}\right)$ substituent constants. 


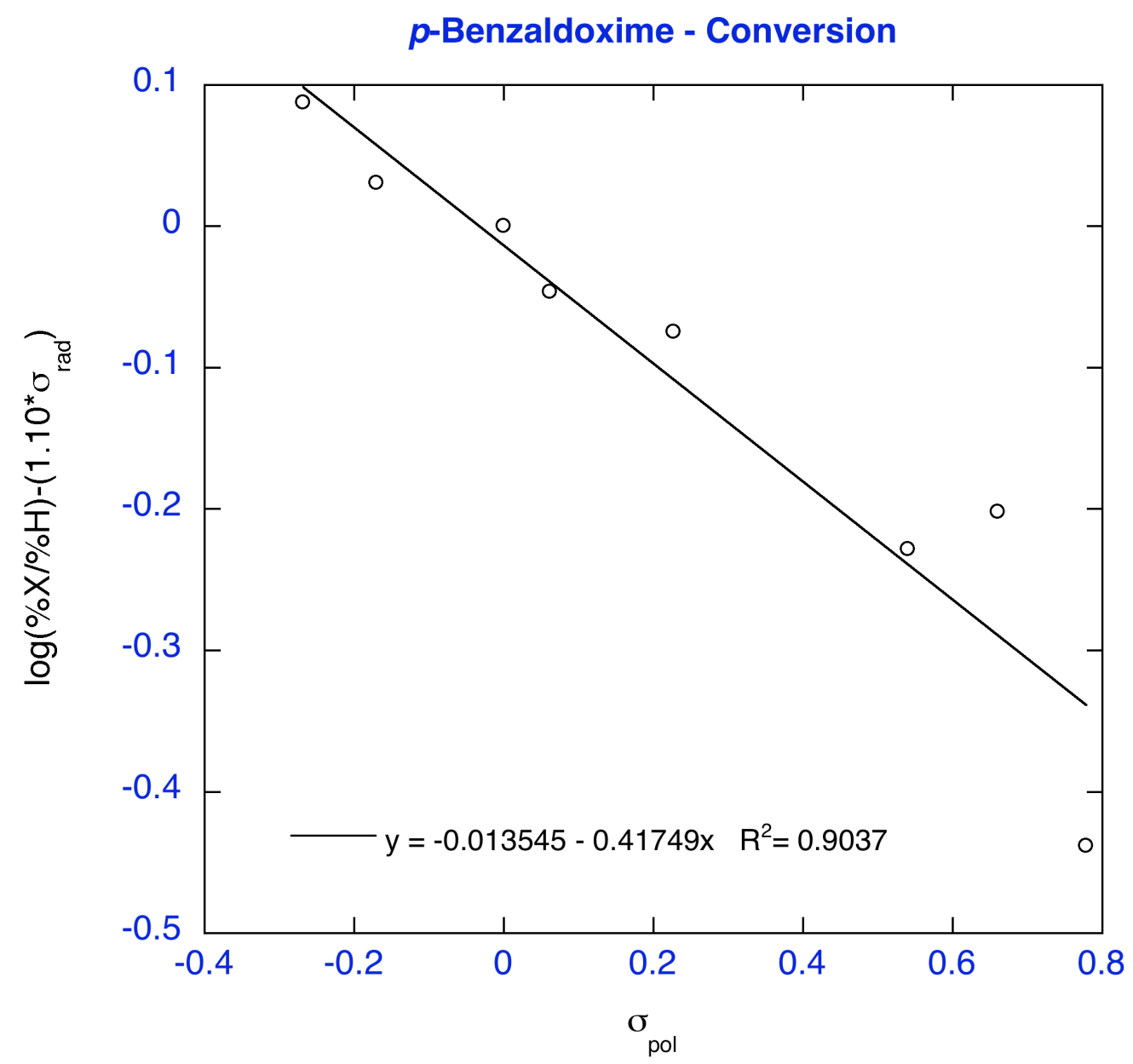

Figure S16. Dual-parameter semilogarithmic plot of the relative conversion of para-substituted benzaldehyde oximes $\left(\%_{\mathrm{X}} / \%_{\mathrm{H}}\right)$ versus radical $\left(\sigma_{\mathrm{rad}}\right)$ and polar $\left(\sigma_{\mathrm{pol}}\right)$ substituent constants. 


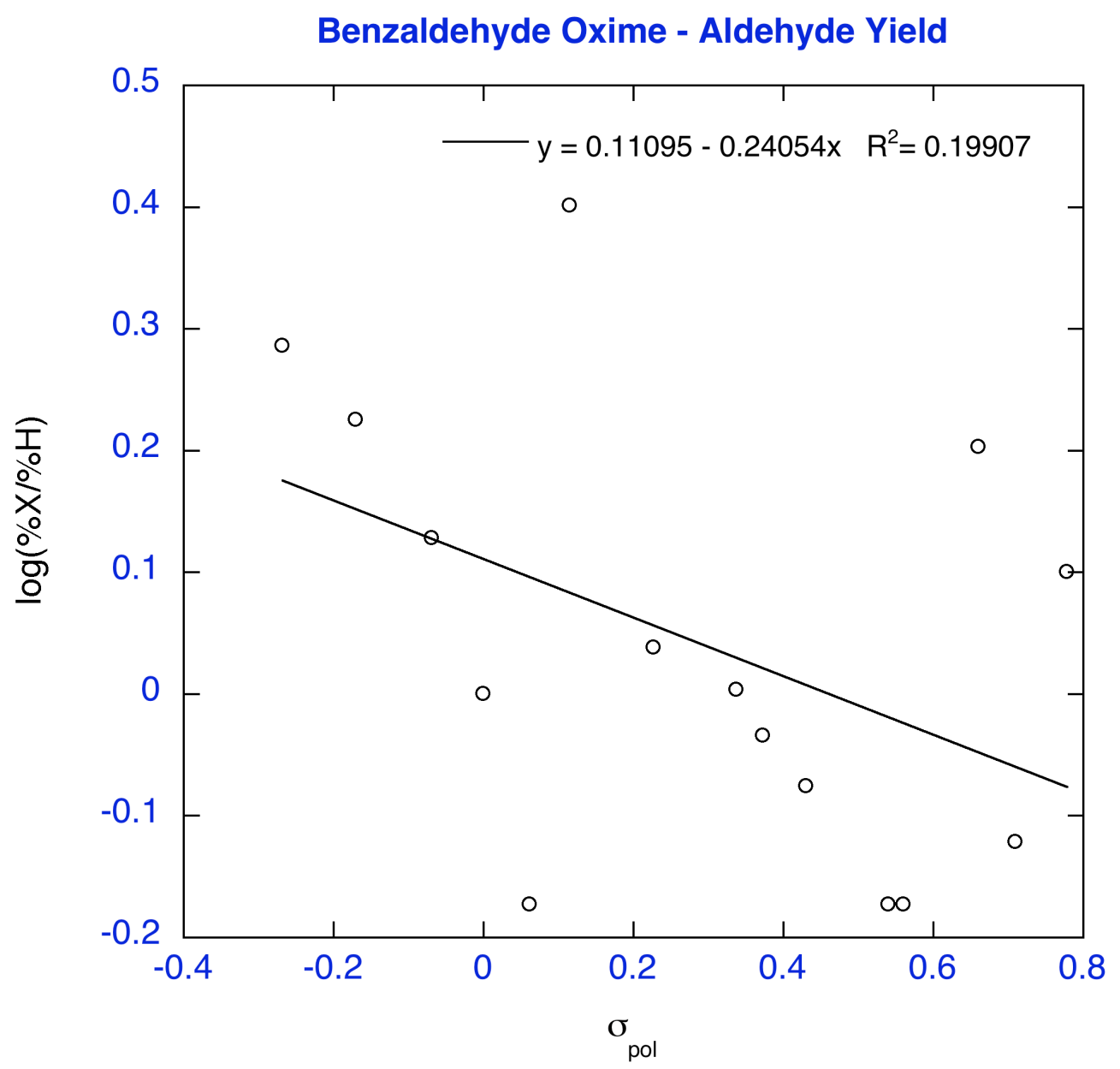

Figure S17. Semilogarithmic plot of the relative aldehyde yield of meta- and para-substituted benzaldehyde oximes $\left(\% \mathrm{x} / \%_{\mathrm{H}}\right)$ versus the polar substituent constant $\sigma_{\text {pol }}$. 


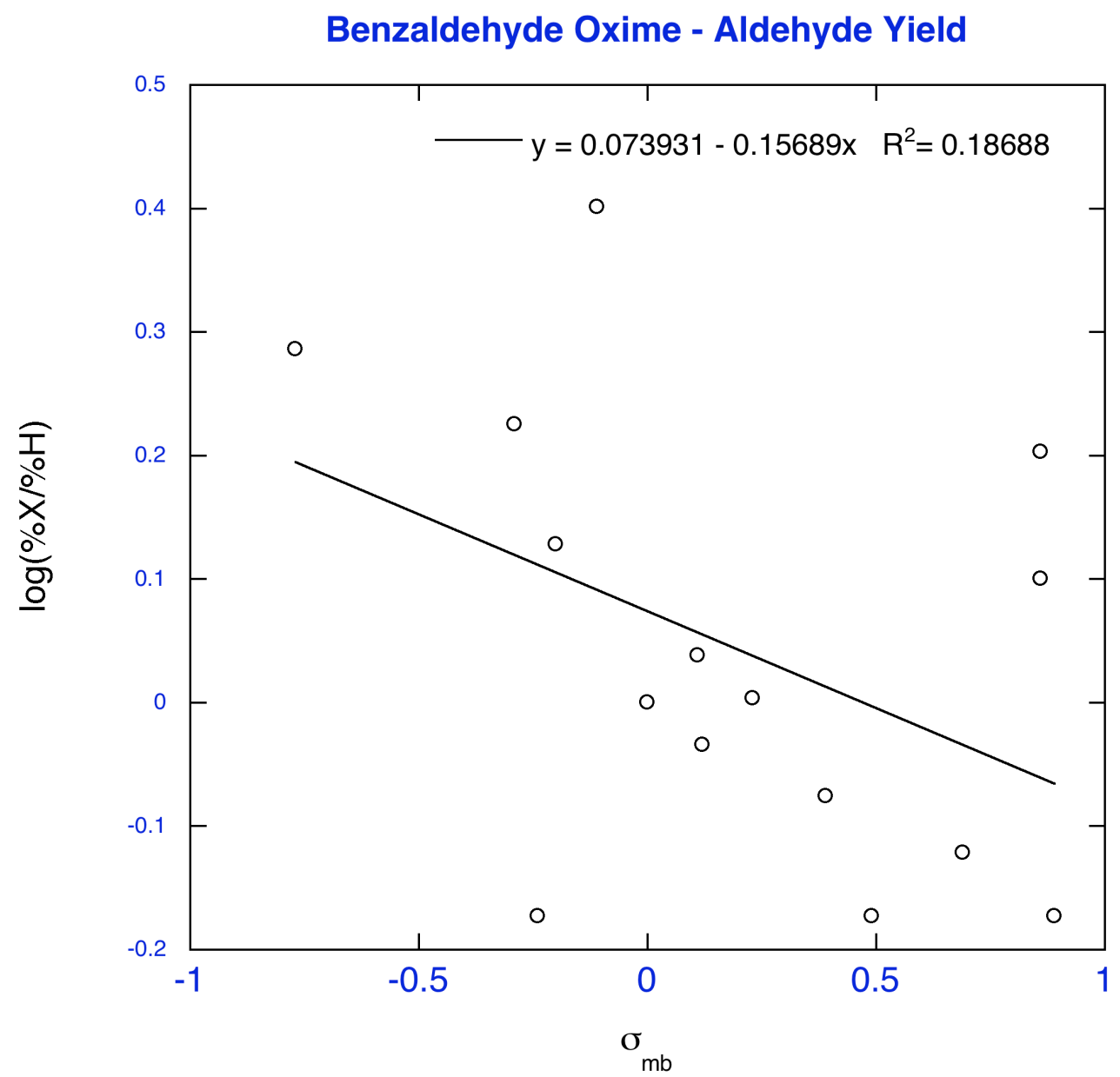

Figure S18. Semilogarithmic plot of the relative aldehyde yield of meta- and para-substituted benzaldehyde oximes $\left(\% \mathrm{x} / \%_{\mathrm{H}}\right)$ versus the polar substituent constant $\sigma_{\mathrm{mb}}$. 


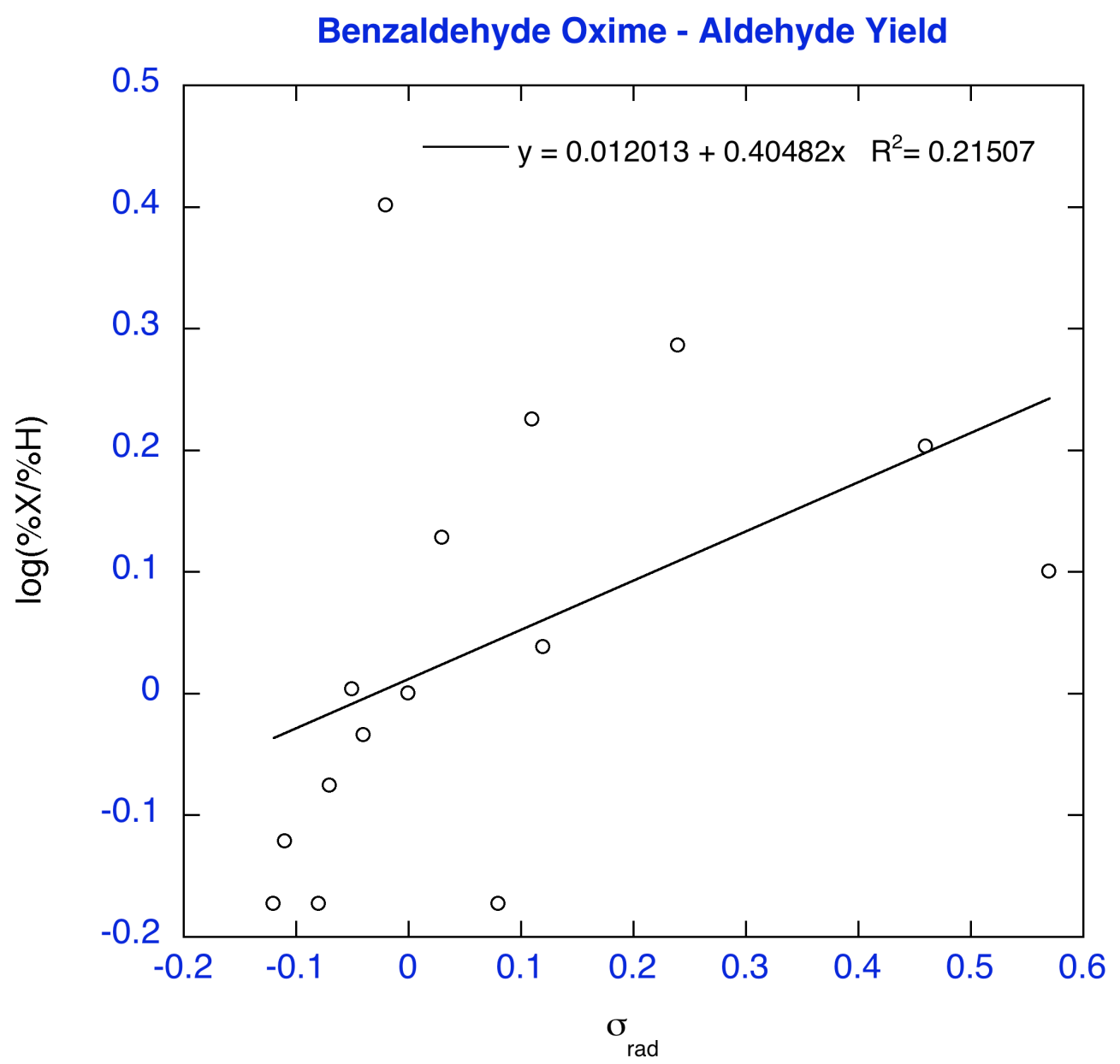

Figure S19. Semilogarithmic plot of the relative aldehyde yield of meta- and para-substituted benzaldehyde oximes $\left(\%_{\mathrm{X}} / \%_{\mathrm{H}}\right)$ versus the radical substituent constant $\sigma_{\text {rad. }}$. 


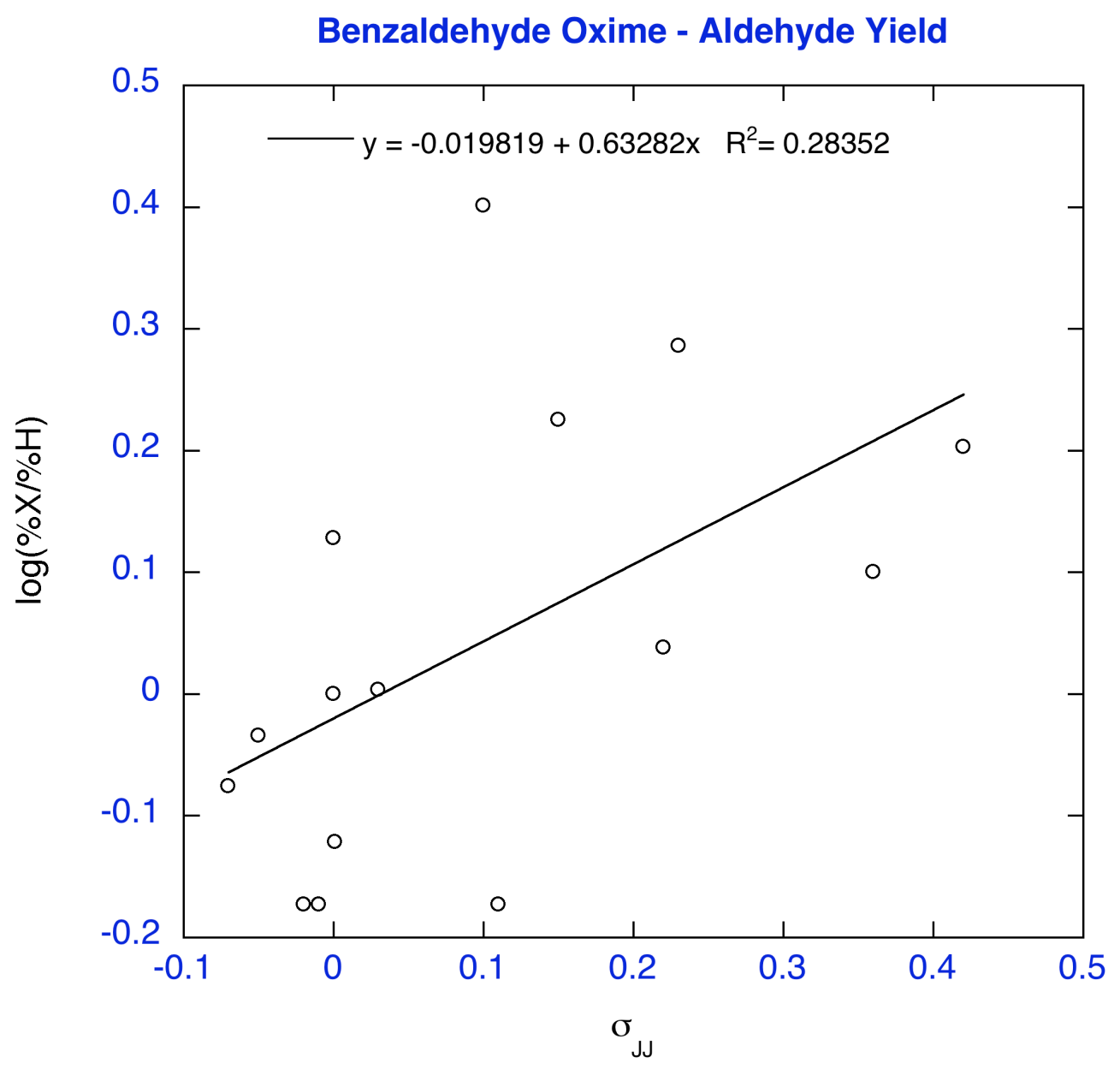

Figure S20. Semilogarithmic plot of the relative aldehyde yield of meta- and para-substituted benzaldehyde oximes $\left(\% \mathrm{X} / \%_{\mathrm{H}}\right)$ versus the radical substituent constant $\sigma_{\mathrm{JJ}}$. 


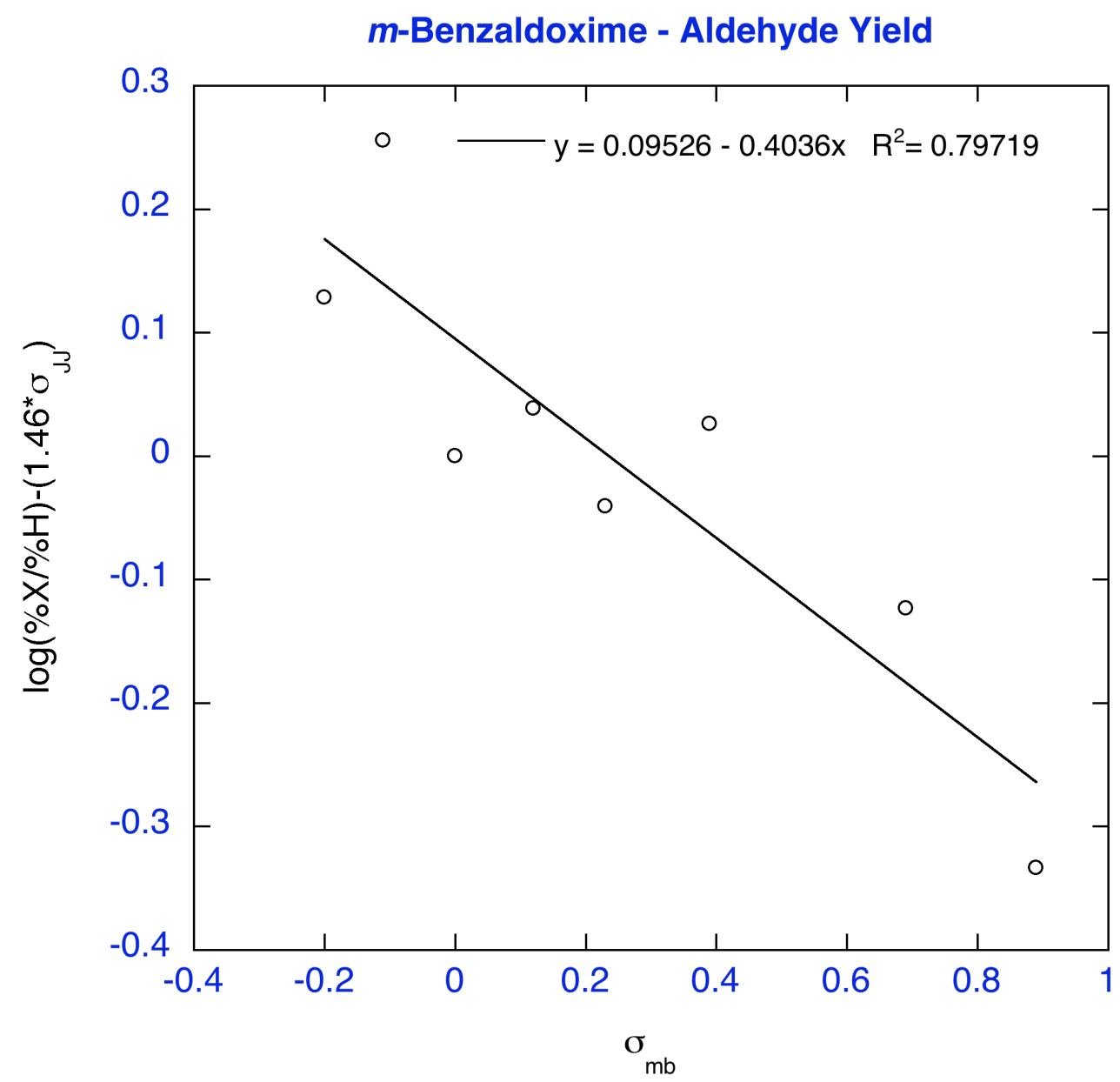

Figure S21. Dual-parameter semilogarithmic plot of the relative aldehyde yield of meta-substituted benzaldehyde oximes $\left(\% \mathrm{X} / \%_{\mathrm{H}}\right)$ versus radical $\left(\sigma_{\mathrm{JJ}}\right)$ and polar $\left(\sigma_{\mathrm{mb}}\right)$ substituent constants. 


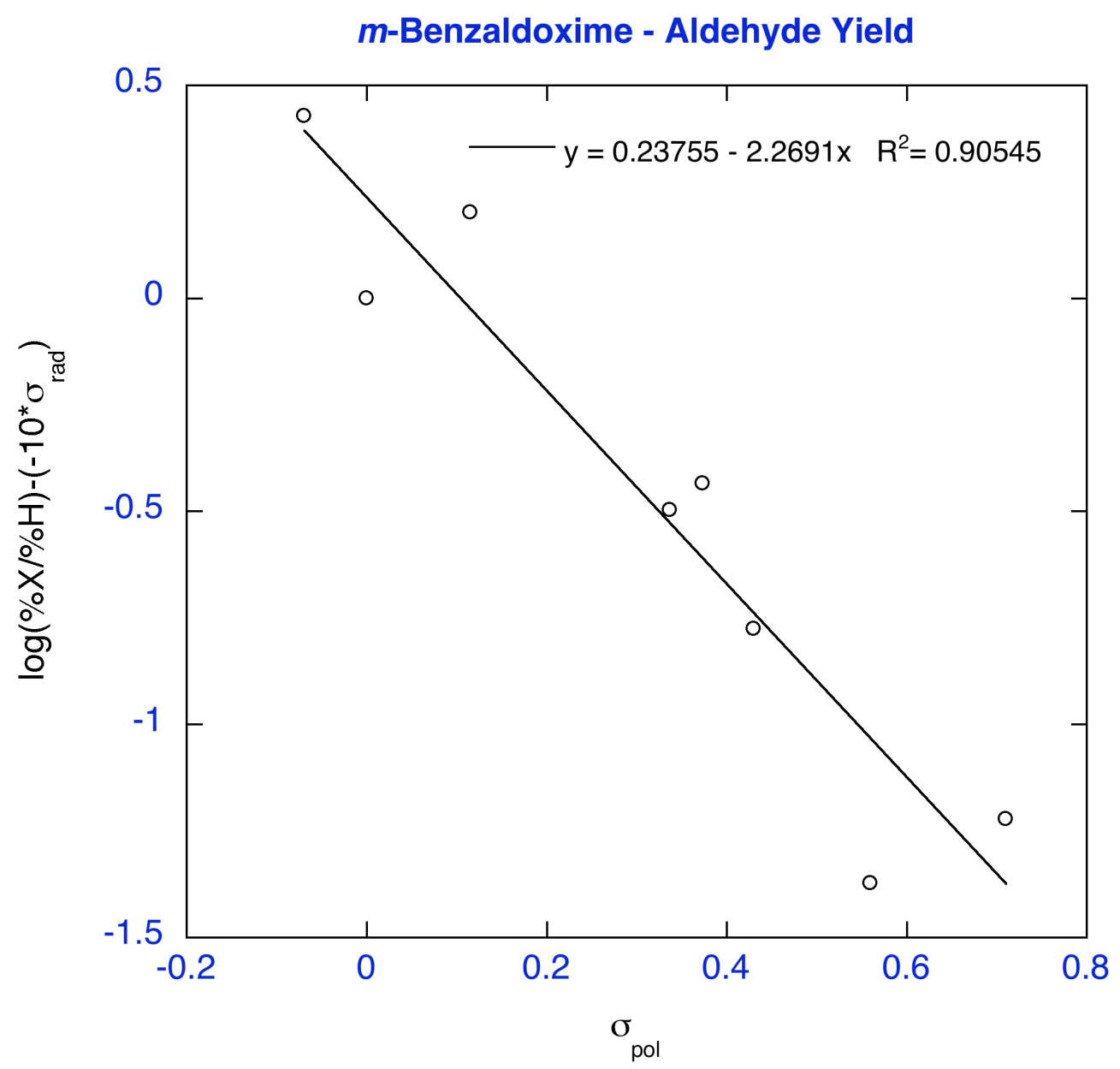

Figure S22. Dual-parameter semilogarithmic plot of the relative aldehyde yield of meta-substituted benzaldehyde oximes $\left(\%_{\mathrm{X}} / \%_{\mathrm{H}}\right)$ versus radical $\left(\sigma_{\mathrm{rad}}\right)$ and polar $\left(\sigma_{\mathrm{pol}}\right)$ substituent constants. 


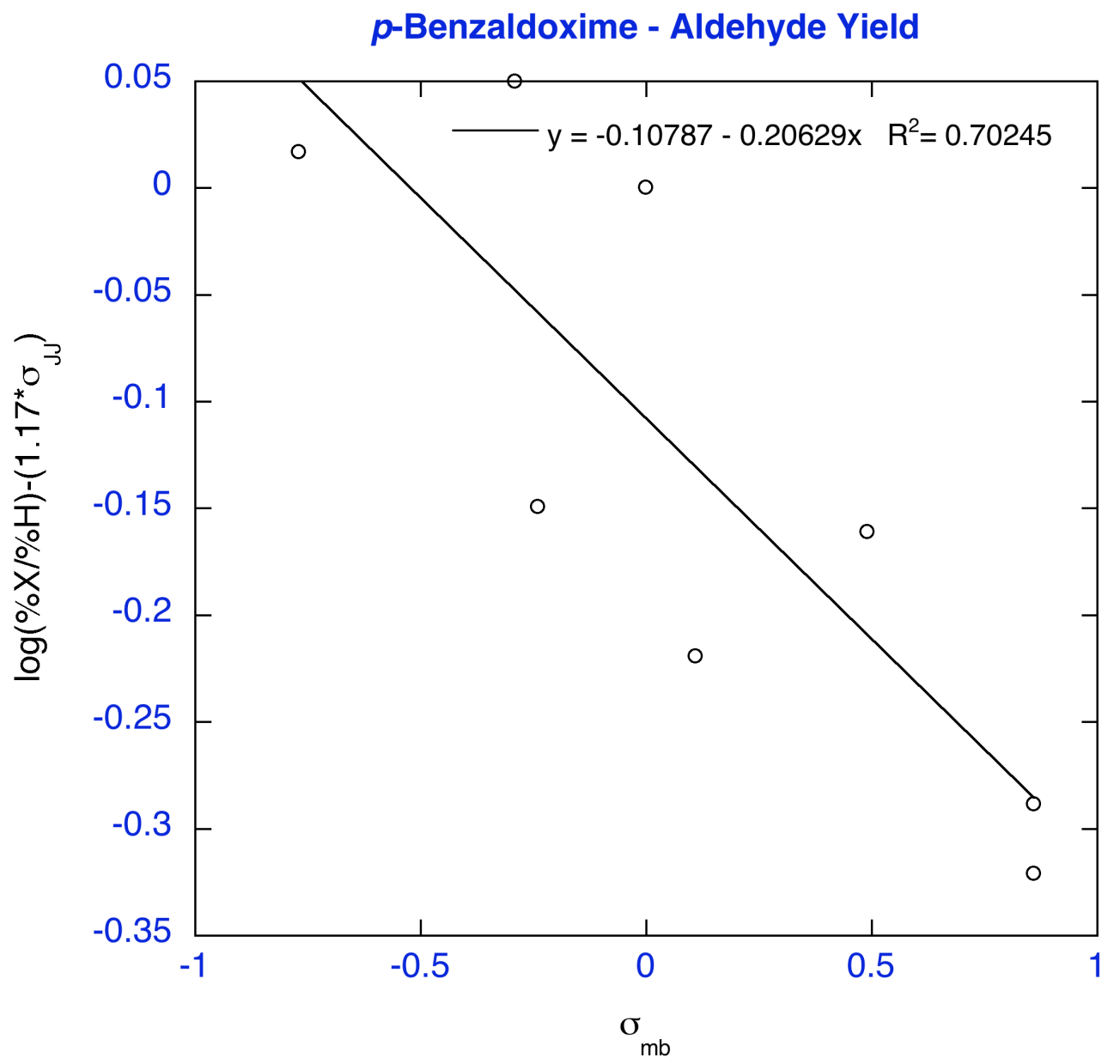

Figure S23. Dual-parameter semilogarithmic plot of the relative aldehyde yield of para-substituted benzaldehyde oximes $\left(\%_{\mathrm{X}} / \%_{\mathrm{H}}\right)$ versus radical $\left(\sigma_{\mathrm{JJ}}\right)$ and polar $\left(\sigma_{\mathrm{mb}}\right)$ substituent constants. 


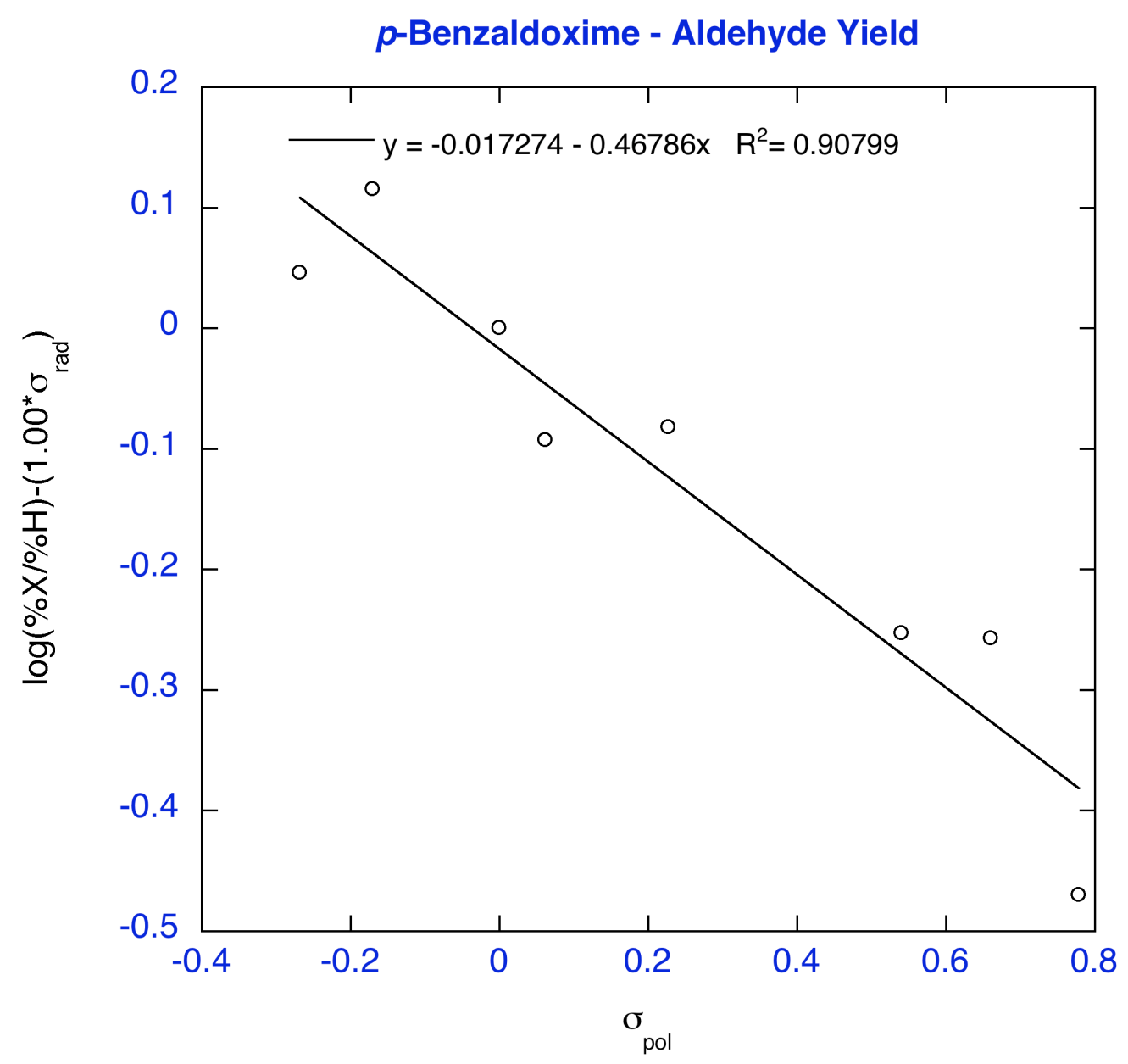

Figure S24. Dual-parameter semilogarithmic plot of the relative aldehyde yield of para-substituted benzaldehyde oximes $\left(\%_{\mathrm{x}} / \%_{\mathrm{H}}\right)$ versus radical $\left(\sigma_{\mathrm{rad}}\right)$ and polar $\left(\sigma_{\mathrm{pol}}\right)$ substituent constants. 


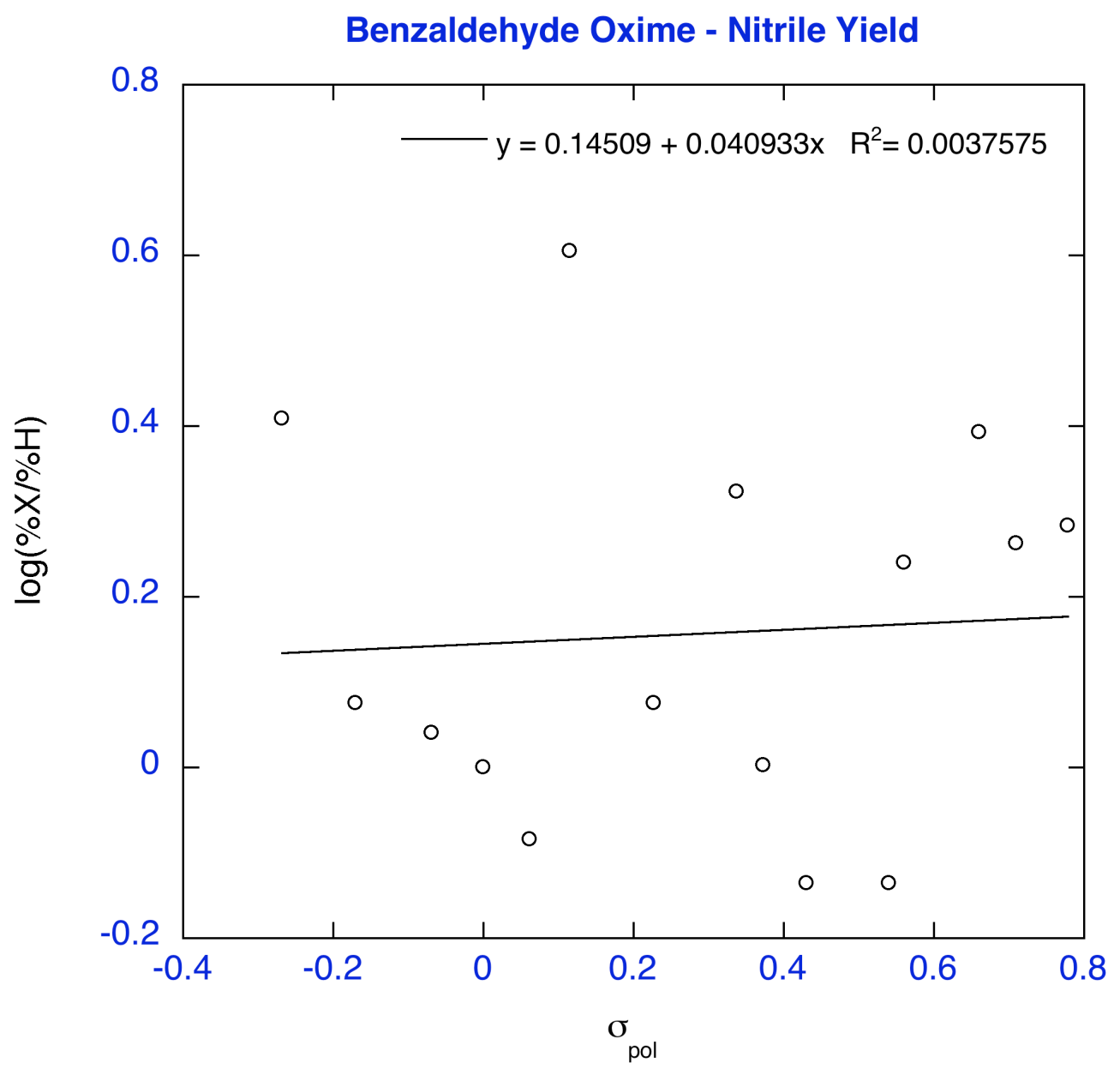

Figure S25. Semilogarithmic plot of the relative nitrile yield of meta- and para-substituted benzaldehyde oximes $\left(\% \mathrm{x} / \%_{\mathrm{H}}\right)$ versus the polar substituent constant $\sigma_{\text {pol }}$. 


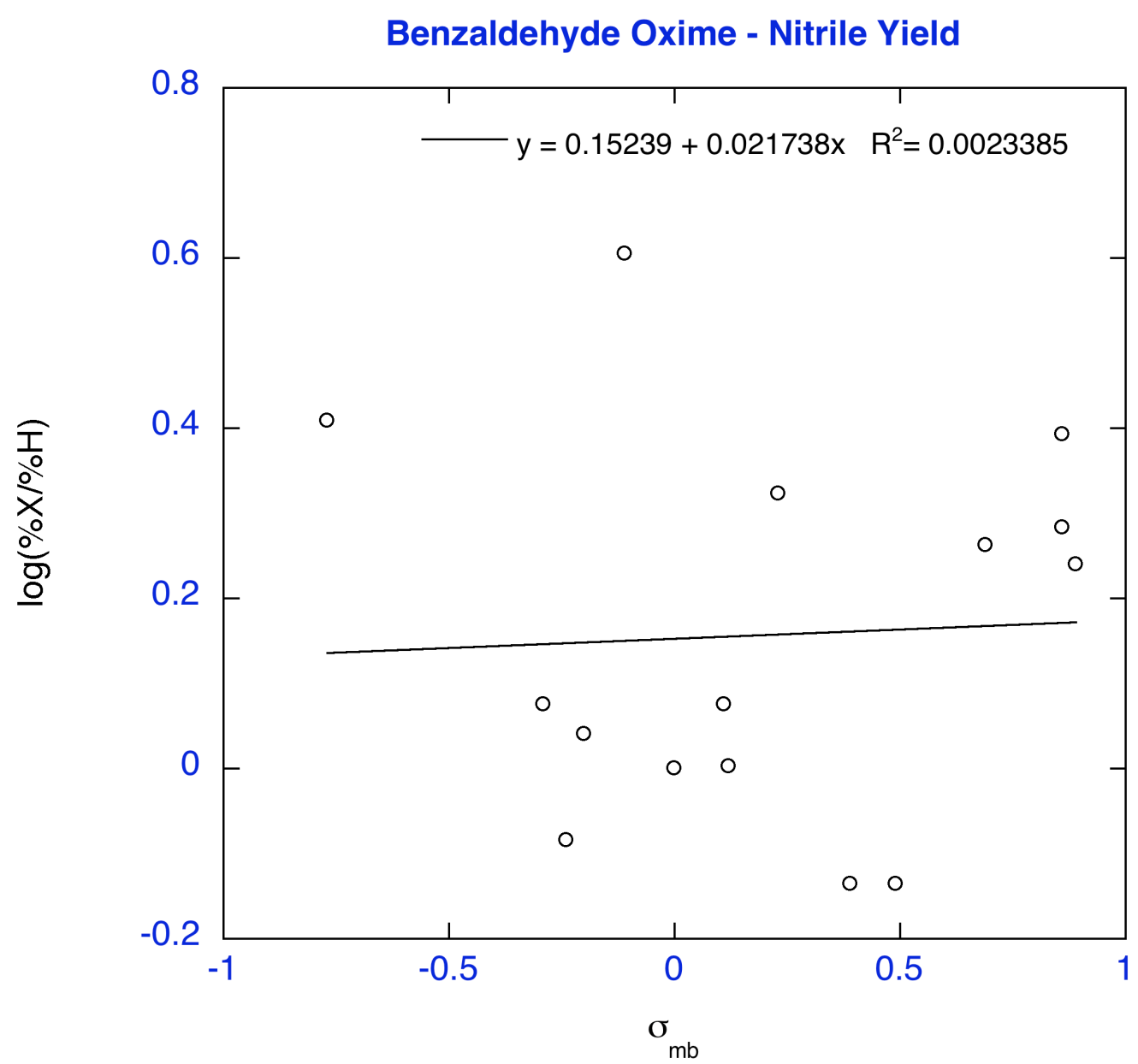

Figure S26. Semilogarithmic plot of the relative nitrile yield of meta- and para-substituted benzaldehyde oximes $\left(\% \mathrm{x} / \%_{\mathrm{H}}\right)$ versus the polar substituent constant $\sigma_{\mathrm{mb}}$. 


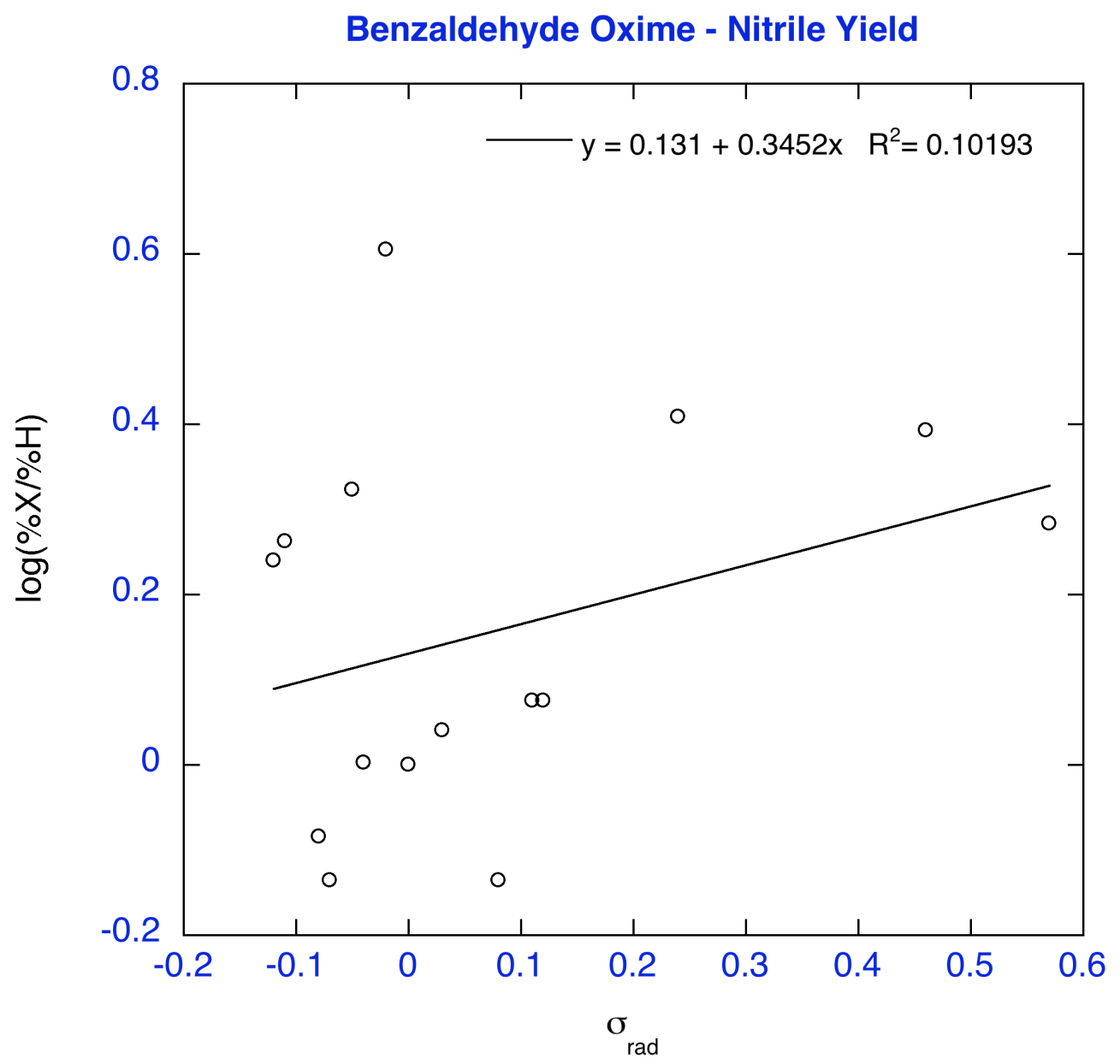

Figure S27. Semilogarithmic plot of the relative nitrile yield of meta- and para-substituted benzaldehyde oximes $\left(\%_{\mathrm{X}} / \%_{\mathrm{H}}\right)$ versus the radical substituent constant $\sigma_{\text {rad. }}$. 


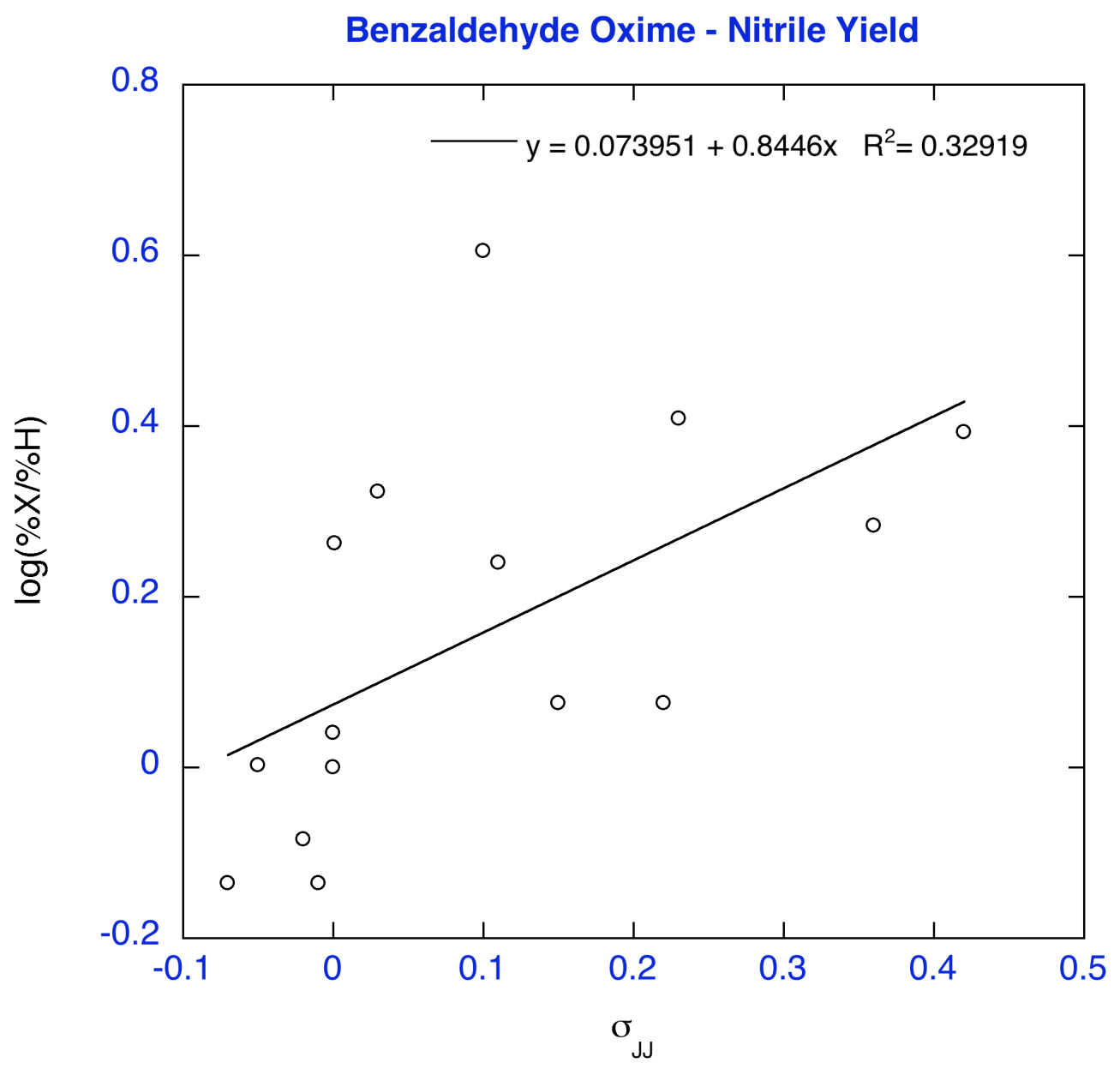

Figure S28. Semilogarithmic plot of the relative nitrile yield of meta- and para-substituted benzaldehyde oximes $\left(\% \mathrm{X} / \%_{\mathrm{H}}\right)$ versus the radical substituent constant $\sigma_{\mathrm{JJ}}$. 


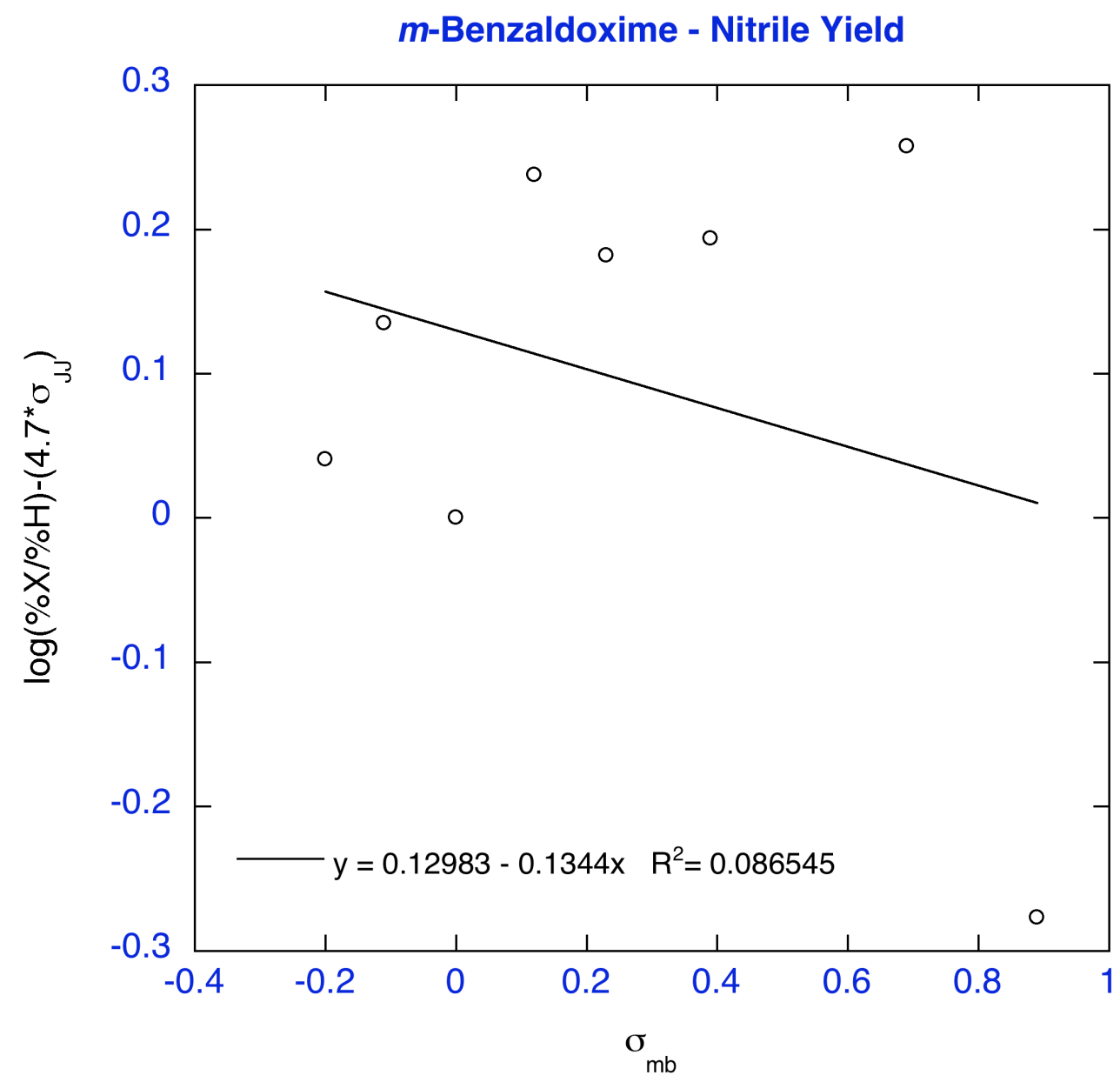

Figure S29. Dual-parameter semilogarithmic plot of the relative nitrile yield of meta-substituted benzaldehyde oximes $\left(\% \mathrm{X} / \%_{\mathrm{H}}\right)$ versus radical $\left(\sigma_{\mathrm{JJ}}\right)$ and polar $\left(\sigma_{\mathrm{mb}}\right)$ substituent constants. 


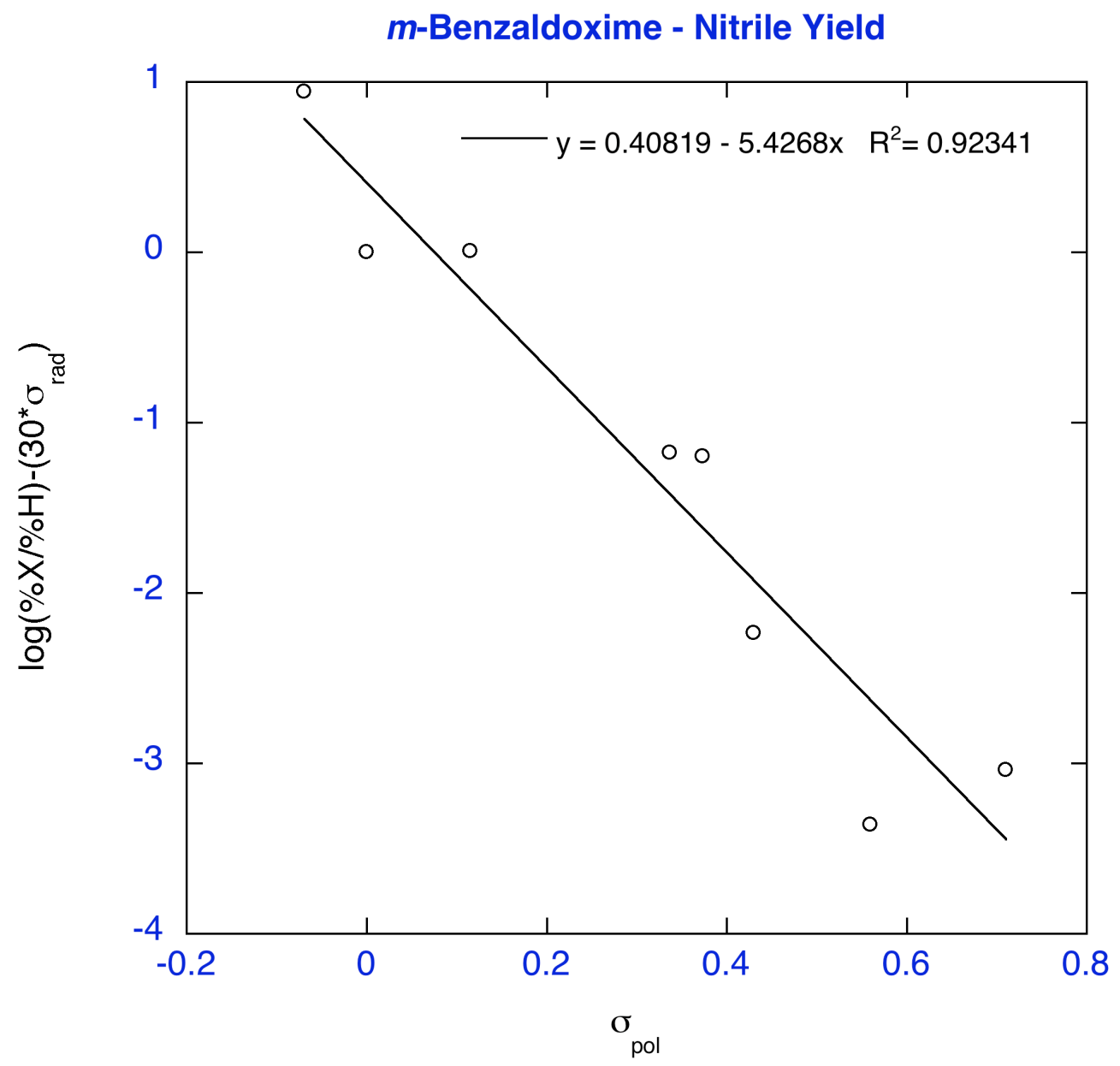

Figure S30. Dual-parameter semilogarithmic plot of the relative nitrile yield of meta-substituted benzaldehyde oximes $\left(\%_{\mathrm{X}} / \%_{\mathrm{H}}\right)$ versus radical $\left(\sigma_{\mathrm{rad}}\right)$ and polar $\left(\sigma_{\mathrm{pol}}\right)$ substituent constants. 


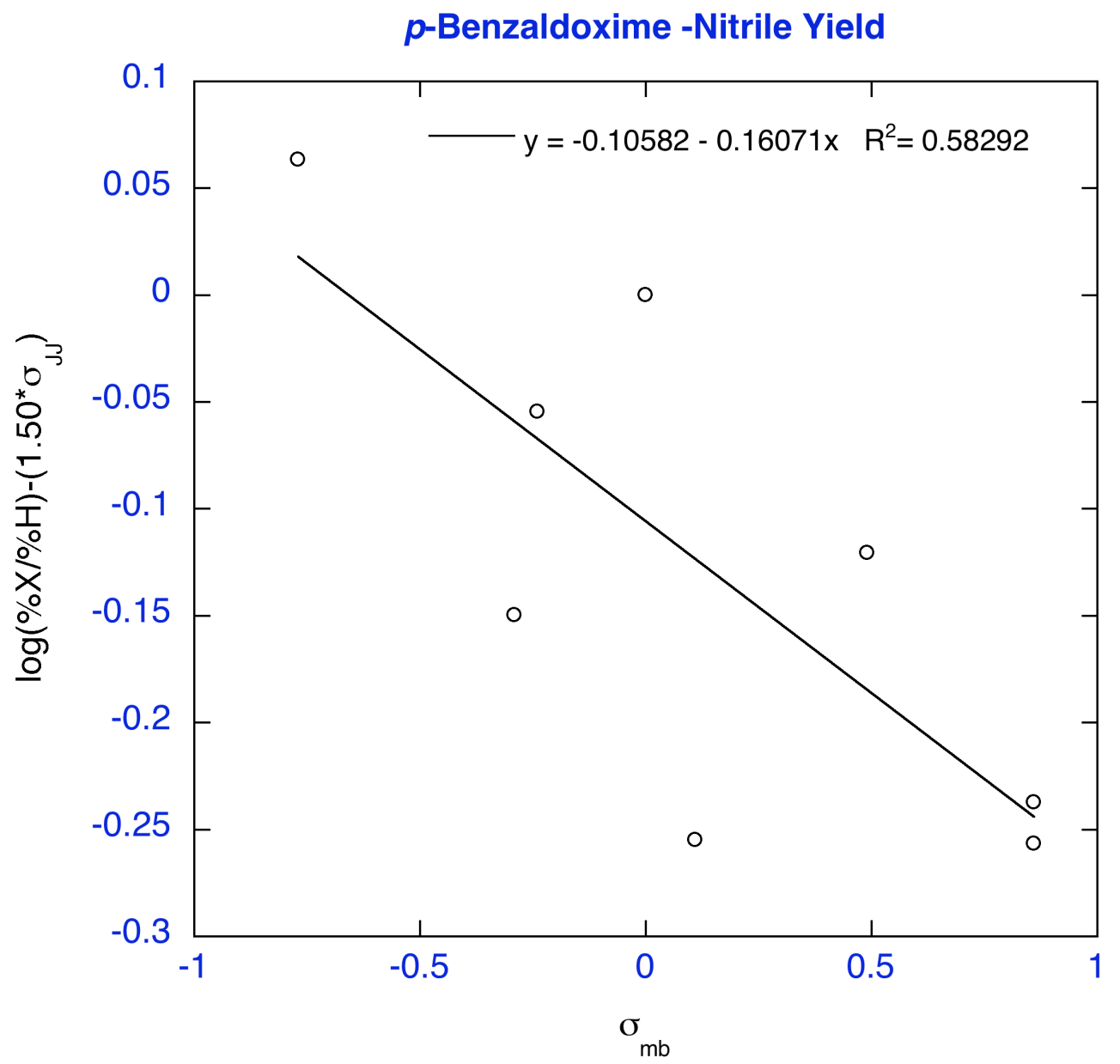

Figure S31. Dual-parameter semilogarithmic plot of the relative nitrile yield of para-substituted benzaldehyde oximes $\left(\%_{\mathrm{X}} / \%_{\mathrm{H}}\right)$ versus radical $\left(\sigma_{\mathrm{JJ}}\right)$ and polar $\left(\sigma_{\mathrm{mb}}\right)$ substituent constants. 


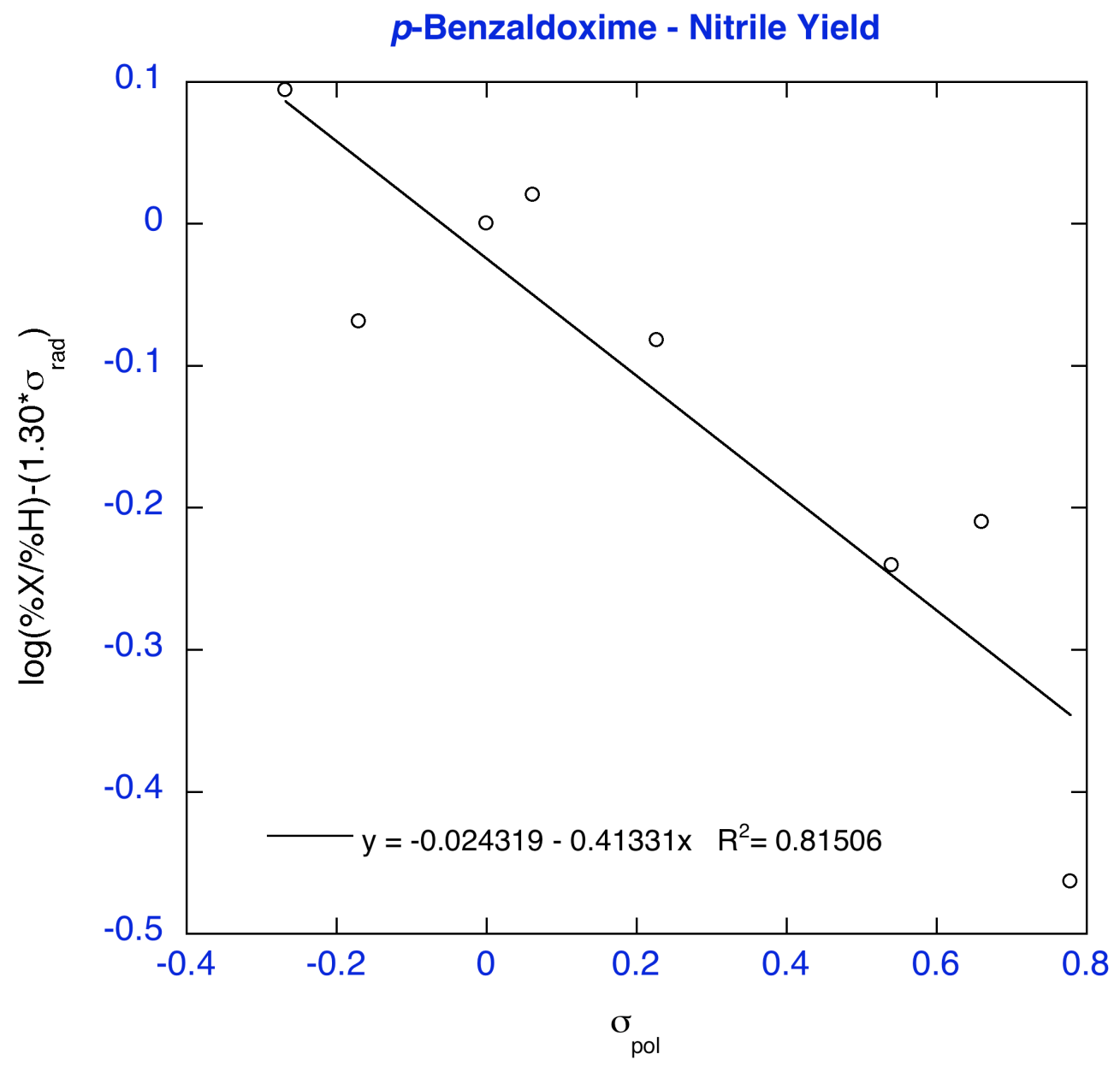

Figure S32. Dual-parameter semilogarithmic plot of the relative nitrile yield of para-substituted benzaldehyde oximes $\left(\% \mathrm{x} / \%_{\mathrm{H}}\right)$ versus radical $\left(\sigma_{\mathrm{rad}}\right)$ and polar $\left(\sigma_{\mathrm{pol}}\right)$ substituent constants. 


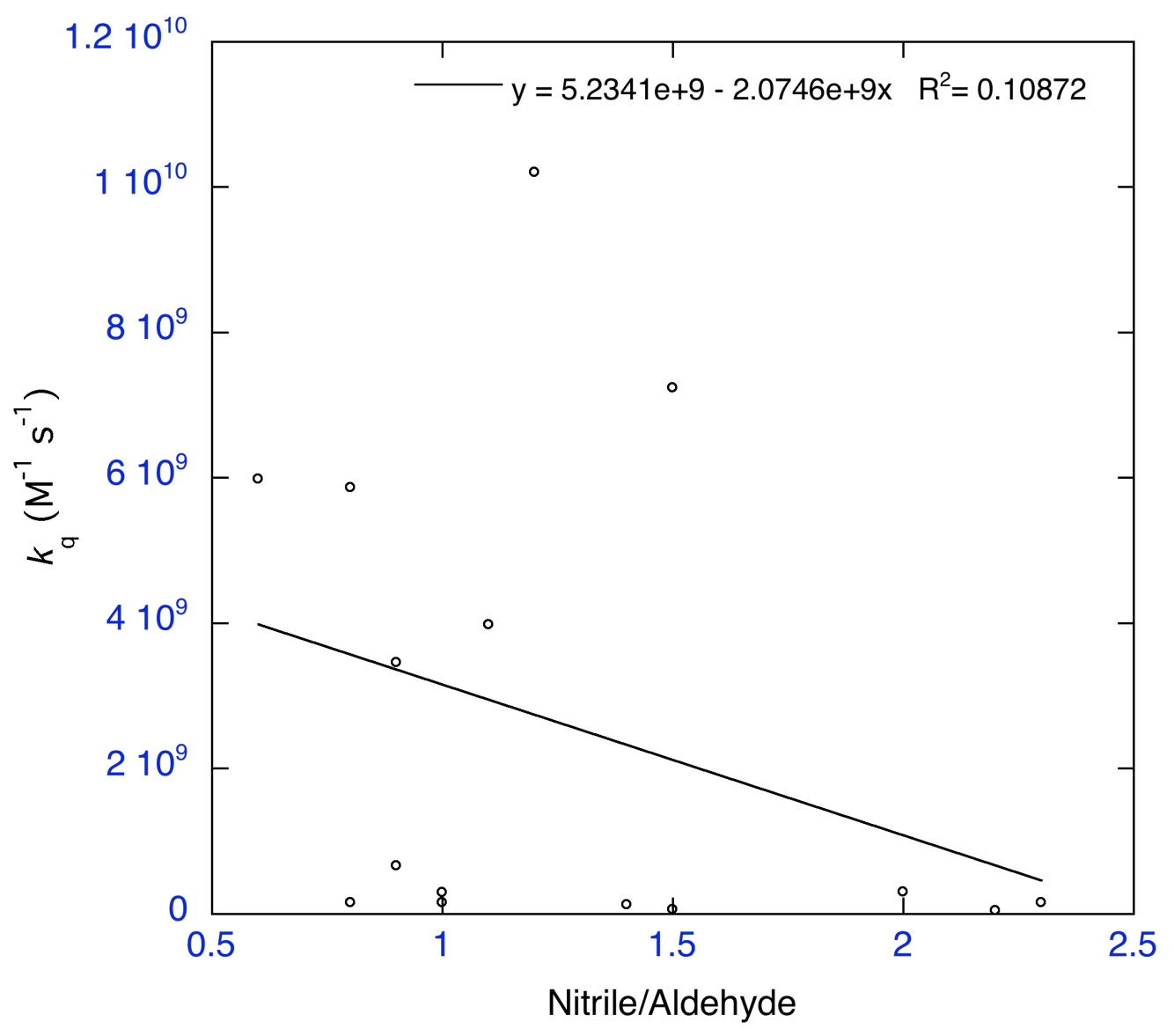

Figure S33. Plot of quenching rate $\left(k_{\mathrm{q}}\right)$ against the nitrile-aldehyde ratio. 


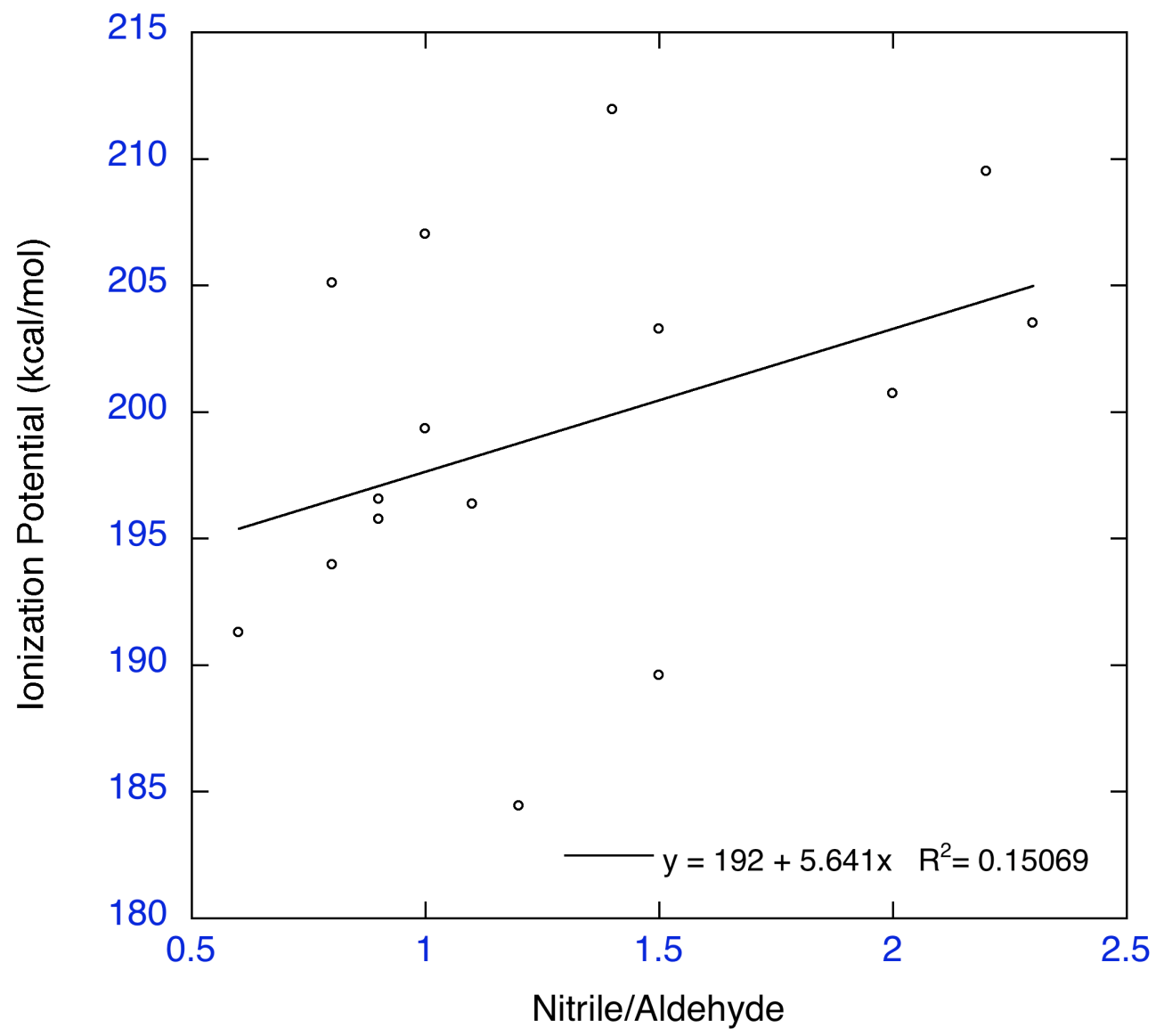

Figure S34. Plot of ionization potential (IP) against the nitrile-aldehyde ratio. 
Table S1. Cartesian coordinates (̊̊) for 2A neutral.

\begin{tabular}{|c|c|c|c|c|}
\hline & Atom & $\mathrm{X}$ & $\mathrm{Y}$ & Z \\
\hline 1 & C7 & .0082566 & 1.8514008 & -.9287307 \\
\hline 2 & N2 & .0679827 & 2.0376717 & -2.2088180 \\
\hline 3 & $\mathrm{C} 2$ & -.0033944 & .6022310 & -.1641868 \\
\hline 4 & 01 & .1316776 & .9515457 & -3.0268395 \\
\hline 5 & $\mathrm{H} 1$ & .1242303 & .1302820 & -2.4619936 \\
\hline $6 \mathrm{H}$ & $\mathrm{H} 3$ & -.0402781 & 2.7773891 & -.3598341 \\
\hline $7 \mathrm{C}$ & $\mathrm{C} 3$ & -.0252885 & -1.7740022 & 1.2265382 \\
\hline $8 N$ & N1 & .0573891 & -.5650636 & -.8465964 \\
\hline 9 & $\mathrm{C} 5$ & -.0766837 & .6277743 & 1.2393136 \\
\hline 10 & $\mathrm{C} 6$ & -.0870565 & -.5731728 & 1.9377477 \\
\hline 11 & $\mathrm{C} 8$ & .0459569 & -1.7137816 & -.1633994 \\
\hline 12 & $\mathrm{H} 4$ & -.1244275 & 1.5794833 & 1.7598332 \\
\hline 13 & H5 & -.1429145 & -.5764035 & 3.0228538 \\
\hline 14 & H6 & .0962653 & -2.6220229 & -.7596723 \\
\hline 15 & $\mathrm{H} 7$ & -.0317154 & -2.7333315 & 1.7337841 \\
\hline
\end{tabular}

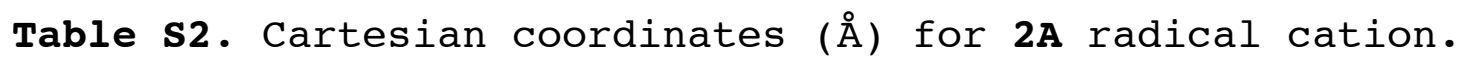

\begin{tabular}{|c|c|c|c|c|}
\hline & Atom & $\mathrm{x}$ & $\mathrm{Y}$ & $\mathrm{Z}$ \\
\hline 1 & $\mathrm{C} 7$ & .0074860 & 1.9387768 & -.9266015 \\
\hline $2 \mathrm{~N}$ & $\mathrm{~N} 2$ & .0752609 & 1.9916900 & -2.2197048 \\
\hline $3 \mathrm{C}$ & $\mathrm{C} 2$ & -.0043170 & .6910124 & -.1815235 \\
\hline 0 & 01 & .1410444 & 1.1440643 & -3.0920285 \\
\hline $\mathrm{H}$ & $\mathrm{H} 1$ & .1089455 & -.4767323 & -1.8638286 \\
\hline $\mathrm{H}$ & H3 & -.0424427 & 2.8845943 & -.4027642 \\
\hline $\mathrm{C}$ & C3 & -.0242275 & -1.7643776 & 1.1534338 \\
\hline $\mathrm{N}$ & $\mathrm{N} 1$ & .0555813 & -.4989815 & -.8442626 \\
\hline $9 \mathrm{C}$ & $\mathrm{C} 5$ & -.0774568 & .6572771 & 1.2152702 \\
\hline 10 & C6 & -.0866955 & -.5656875 & 1.8784414 \\
\hline 11 & $\mathrm{C} 8$ & .0474345 & -1.7026425 & -.2240012 \\
\hline 12 & $\mathrm{H} 4$ & -.1263645 & 1.5915271 & 1.7636722 \\
\hline 13 & H5 & -.1430294 & -.5888158 & 2.9623488 \\
\hline 14 & H6 & .0994315 & -2.5733658 & -.8670005 \\
\hline 15 & $\mathrm{H} 7$ & -.0306508 & -2.7283389 & 1.6485489 \\
\hline
\end{tabular}


Table s3. Cartesian coordinates ( $)$ for 2 B neutral.

\begin{tabular}{|c|c|c|c|c|}
\hline & Atom & $\mathrm{X}$ & $\mathrm{Y}$ & $\mathrm{Z}$ \\
\hline $1 \mathrm{H}$ & $\mathrm{H} 1$ & 1.9032108 & -1.7202509 & .0000000 \\
\hline 2 & $\mathrm{C} 1$ & 1.6466106 & -.6599300 & .0000000 \\
\hline $3 \mathrm{~N}$ & $\mathrm{~N} 1$ & 2.5423620 & .2560365 & .0000000 \\
\hline & $\mathrm{C} 2$ & .2162937 & -.3188203 & .0000000 \\
\hline 50 & 01 & 3.8256943 & -.2959942 & .0000000 \\
\hline & $\mathrm{H} 2$ & 4.3940920 & .4904050 & .0000000 \\
\hline $7 \mathrm{C}$ & $\mathrm{C} 3$ & -2.4807151 & .1499148 & .0000000 \\
\hline 8 & $\mathrm{C} 4$ & -.2406243 & 1.0091539 & .0000000 \\
\hline $9 \mathrm{~N}$ & $\mathrm{~N} 2$ & -.6159066 & -1.3764758 & .0000000 \\
\hline 10 & $\mathrm{C} 6$ & -1.9301251 & -1.1319195 & .0000000 \\
\hline 110 & $\mathrm{C} 7$ & -1.6102020 & 1.2419953 & .0000000 \\
\hline $12 \mathrm{~F}$ & H3 & .4784807 & 1.8212191 & .0000000 \\
\hline $13 \mathrm{~F}$ & H5 & -2.5759234 & -2.0086962 & .0000000 \\
\hline $14 \mathrm{~F}$ & H6 & -1.9950637 & 2.2584187 & .0000000 \\
\hline 15 & $\mathrm{H} 7$ & -3.5581840 & .2849438 & .0000000 \\
\hline
\end{tabular}

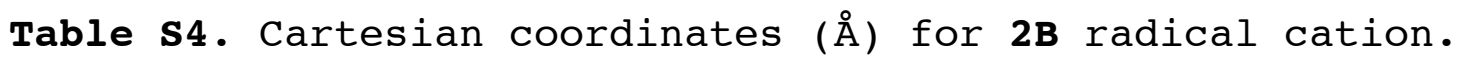

\begin{tabular}{|c|c|c|c|c|c|}
\hline & & Atom & $\mathrm{x}$ & $\mathrm{Y}$ & $\mathrm{Z}$ \\
\hline $1 \mathrm{H}$ & & $\mathrm{H} 1$ & 1.9485127 & -1.6870302 & .0000000 \\
\hline & $\mathrm{C}$ & $\mathrm{C} 1$ & 1.6292179 & -.6449105 & .0000000 \\
\hline 3 & $\mathrm{~N}$ & $\mathrm{~N} 1$ & 2.5072849 & .3475881 & .0000000 \\
\hline 4 & $\mathrm{C}$ & $\mathrm{C} 2$ & .2376697 & -.3416905 & .0000000 \\
\hline 5 & 0 & 01 & 3.7490825 & -.1341392 & .0000000 \\
\hline 6 & $\mathrm{H}$ & $\mathrm{H} 2$ & 4.3388482 & .6495123 & .0000000 \\
\hline 7 & $\mathrm{C}$ & $\mathrm{C} 3$ & -2.4539061 & .0919921 & .0000000 \\
\hline 8 & $\mathrm{C}$ & $\mathrm{C} 4$ & -.2496909 & 1.0054284 & .0000000 \\
\hline & $\mathrm{N}$ & $\mathrm{N} 2$ & -.5766104 & -1.4335628 & .0000000 \\
\hline 10 & $\mathrm{C}$ & $\mathrm{C} 6$ & -1.8749208 & -1.2156947 & .0000000 \\
\hline 11 & $\mathrm{C}$ & $\mathrm{C} 7$ & -1.6217056 & 1.2123085 & .0000000 \\
\hline 12 & $\mathrm{H}$ & H3 & .4538615 & 1.8308689 & .0000000 \\
\hline 13 & $\mathrm{H}$ & H5 & -2.5184296 & -2.0925196 & .0000000 \\
\hline 14 & $\mathrm{H}$ & $\mathrm{H} 6$ & -2.0343457 & 2.2157757 & .0000000 \\
\hline 15 & $\mathrm{H}$ & $\mathrm{H} 7$ & -3.5348683 & .1960735 & .0000000 \\
\hline
\end{tabular}


Table S5. Cartesian coordinates (̊) for 2C neutral.

\begin{tabular}{|c|c|c|c|c|}
\hline & Atom & $\mathrm{X}$ & $\mathrm{Y}$ & Z \\
\hline & ----- & ----------- & -ーーー-ーーー-ーー & \\
\hline $1 \mathrm{H}$ & $\mathrm{H} 1$ & 1.9674321 & .9266507 & -1.1455259 \\
\hline $2 \mathrm{C}$ & $\mathrm{C} 1$ & 1.5084305 & -.0278028 & -.8750286 \\
\hline $3 \mathrm{~N}$ & $\mathrm{~N} 1$ & 2.0774367 & -1.1302339 & -1.1962527 \\
\hline $4 \mathrm{C}$ & $\mathrm{C} 2$ & .2347873 & .0071854 & -.1394864 \\
\hline 50 & 01 & 3.2700248 & -.8813235 & -1.8843490 \\
\hline $6 \mathrm{H}$ & $\mathrm{H} 2$ & 3.5939242 & -1.7775165 & -2.0673701 \\
\hline 7 & C3 & -2.1359659 & .1037467 & 1.2353351 \\
\hline $8 \mathrm{~N}$ & $\mathrm{~N} 2$ & -.3556083 & -1.1551484 & .2003326 \\
\hline $9 \mathrm{C}$ & $\mathrm{C} 5$ & -.3185935 & 1.2565207 & .1833093 \\
\hline $10 \mathrm{C}$ & C6 & -1.5224992 & 1.3030490 & .8818013 \\
\hline $11 \mathrm{C}$ & C7 & -1.5064221 & -1.0908085 & .8680092 \\
\hline $12 \mathrm{H}$ & $\mathrm{H} 4$ & .1909210 & 2.1700469 & -.1105295 \\
\hline $13 \mathrm{H}$ & $\mathrm{H} 5$ & -1.9709450 & 2.2576800 & 1.1432348 \\
\hline $14 \mathrm{H}$ & H6 & -1.9575218 & -2.0478955 & 1.1270731 \\
\hline $15 \mathrm{H}$ & $\mathrm{H} 7$ & -3.0754008 & .0858499 & 1.7794465 \\
\hline
\end{tabular}

Table s6. Cartesian coordinates $(\AA)$ for $2 \mathrm{C}$ radical cation.

\begin{tabular}{|c|c|c|c|c|}
\hline & Atom & $\mathrm{x}$ & $\mathrm{Y}$ & $\mathrm{Z}$ \\
\hline 1 & $\mathrm{H} 1$ & 1.9789662 & .9463470 & -1.1455485 \\
\hline 2 & $\mathrm{C} 1$ & 1.4860022 & .0161994 & -.8608616 \\
\hline 3 & $\mathrm{~N} 1$ & 2.0457768 & -1.1471010 & -1.1803629 \\
\hline 4 & $\mathrm{C} 2$ & .2528525 & .0336426 & -.1503951 \\
\hline 5 & 01 & 3.1850489 & -.9296139 & -1.8346705 \\
\hline 6 & $\mathrm{H} 2$ & 3.5423929 & -1.8201383 & -2.0403026 \\
\hline 7 & $\mathrm{C} 3$ & -2.1047369 & .0987522 & 1.2178931 \\
\hline & N2 & -.3196573 & -1.1603497 & .1797667 \\
\hline $9 \mathrm{C}$ & $\therefore \quad \mathrm{C} 5$ & -.3235345 & 1.2984897 & .1843919 \\
\hline & $\mathrm{C} 6$ & -1.5231808 & 1.3219323 & .8812585 \\
\hline 11 & C7 & -1.4548399 & -1.1181256 & .8389776 \\
\hline 12 & $\mathrm{H} 4$ & .1777688 & 2.2173302 & -.1054698 \\
\hline 13 & H5 & -1.9934294 & 2.2606249 & 1.1549949 \\
\hline 14 & H6 & -1.9059318 & -2.0732849 & 1.0982903 \\
\hline 15 & $\mathrm{H} 7$ & -3.0434975 & .0552949 & 1.7620378 \\
\hline
\end{tabular}


Table s7. Cartesian coordinates (̊̊) for 3A neutral.

\begin{tabular}{rllrrr} 
& & Atom & \multicolumn{1}{c}{ X } & Y \\
1 & C & C2 & -.3902739 & -.0102710 & .1669648 \\
2 & C & C1 & -.9436737 & .0545583 & 1.5026637 \\
3 & N & N1 & -.3832061 & .1629366 & 2.6604620 \\
4 & H & H3 & -2.0291986 & .0046315 & 1.5503256 \\
5 & O & O2 & .9769446 & .0364701 & -.0086445 \\
6 & C & C4 & 1.2186976 & -.0385642 & -1.3512707 \\
7 & C & C5 & .0462662 & -.1322871 & -2.0360267 \\
8 & C & C6 & -.9956287 & -.1150085 & -1.0591190 \\
9 & H & H6 & 2.2576239 & -.0127996 & -1.6414477 \\
10 & H & H9 & -.0640966 & -.2058666 & -3.1087202 \\
11 & H & H10 & -2.0604356 & -.1726736 & -1.2367869 \\
12 & O & O1 & .9906750 & .2362340 & 2.7300950 \\
13 & H & H1 & 1.3763059 & .1926403 & 1.8315045
\end{tabular}

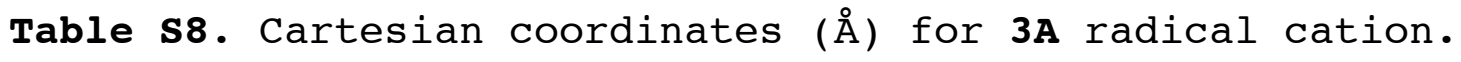

\begin{tabular}{|c|c|c|c|c|}
\hline & Atom & $\mathrm{X}$ & $\mathrm{Y}$ & Z \\
\hline 1 & $\mathrm{C} 2$ & -.3868153 & -.0076172 & .1787089 \\
\hline 2 & $\mathrm{C} 1$ & -.9378608 & .0545890 & 1.4641467 \\
\hline 3 & $\mathrm{~N} 1$ & -.3499807 & .1630944 & 2.6609612 \\
\hline 4 & H3 & -2.0206949 & .0102021 & 1.5392971 \\
\hline 5 & $\mathrm{O} 2$ & .9814351 & .0411802 & .0002151 \\
\hline 6 & $\mathrm{C} 4$ & 1.2116368 & -.0352489 & -1.3288188 \\
\hline 7 & $\mathrm{C} 5$ & .0180363 & -.1350348 & -2.0354842 \\
\hline 8 & $\mathrm{C} 6$ & -1.0030284 & -.1185834 & -1.0877751 \\
\hline 9 & H6 & 2.2493287 & -.0091613 & -1.6346868 \\
\hline 10 & $\mathrm{H} 9$ & -.0753604 & -.2100810 & -3.1103546 \\
\hline 11 & $\mathrm{H} 10$ & -2.0699575 & -.1782453 & -1.2609784 \\
\hline 12 & 01 & .9623081 & .2307182 & 2.7445974 \\
\hline 13 & $\mathrm{H} 1$ & 1.4209529 & .1941878 & 1.8701715 \\
\hline
\end{tabular}


Table s9. Cartesian coordinates (̊̊) for 3B neutral.

\begin{tabular}{|c|c|c|c|c|c|}
\hline & & Atom & $\mathrm{X}$ & $\mathrm{Y}$ & $\mathrm{Z}$ \\
\hline 1 & $\mathrm{C}$ & $\mathrm{C} 2$ & .2059664 & -.3157835 & .1760005 \\
\hline 2 & $\mathrm{C}$ & $\mathrm{C} 1$ & .8233740 & -.1515825 & 1.4694875 \\
\hline 3 & $\mathrm{~N}$ & $\mathrm{~N} 1$ & .8538172 & .8799427 & 2.2395160 \\
\hline 4 & $\mathrm{H}$ & $\mathrm{H} 3$ & 1.3455096 & -1.0180277 & 1.8688196 \\
\hline 5 & $\mathrm{O}$ & 01 & .1687841 & 1.9708190 & 1.6792540 \\
\hline 6 & $\mathrm{H}$ & $\mathrm{H} 2$ & .2789994 & 2.6517976 & 2.3607134 \\
\hline 7 & 0 & 02 & .3732495 & -1.5645460 & -.3833578 \\
\hline 8 & $\mathrm{C}$ & $\mathrm{C} 4$ & -.2586800 & -1.5533954 & -1.5824928 \\
\hline 9 & $\mathrm{C}$ & $\mathrm{C} 5$ & -.8312005 & -.3368332 & -1.8163059 \\
\hline 10 & $\mathrm{C}$ & $\mathrm{C} 6$ & -.5329825 & .4687936 & -.6780453 \\
\hline 11 & $\mathrm{H}$ & $\mathrm{H} 7$ & -.2120122 & -2.4735725 & -2.1453756 \\
\hline 12 & $\mathrm{H}$ & $\mathrm{H} 8$ & -1.3974815 & -.0501472 & -2.6914606 \\
\hline 13 & $\mathrm{H}$ & $\mathrm{H} 10$ & -.8173435 & 1.4925350 & -.4967527 \\
\hline
\end{tabular}

Table $\mathbf{5 1 0 .}$. Cartesian coordinates $(\AA)$ for $3 \mathbf{B}$ radical cation.

\begin{tabular}{|c|c|c|c|c|c|}
\hline & & Atom & $\mathrm{X}$ & $\mathrm{Y}$ & $\mathrm{Z}$ \\
\hline & & & & & \\
\hline 1 & $\mathrm{C}$ & $\mathrm{C} 2$ & .2183829 & -.3327340 & .1887530 \\
\hline 2 & $\mathrm{C}$ & $\mathrm{C} 1$ & .8200403 & -.1718670 & 1.4456251 \\
\hline 3 & $\mathrm{~N}$ & N1 & .8369024 & .9033772 & 2.2246454 \\
\hline 4 & $\mathrm{H}$ & H3 & 1.3508299 & -1.0207424 & 1.8672370 \\
\hline 5 & 0 & 01 & .1839169 & 1.9459877 & 1.6937322 \\
\hline 6 & $\mathrm{H}$ & $\mathrm{H} 2$ & .2707956 & 2.6597896 & 2.3581679 \\
\hline 7 & 0 & $\mathrm{O} 2$ & .3779586 & -1.5809944 & -.3812822 \\
\hline 8 & $\mathrm{C}$ & $\mathrm{C} 4$ & -.2462209 & -1.5582433 & -1.5593678 \\
\hline 9 & $\mathrm{C}$ & $\mathrm{C} 5$ & -.8336877 & -.3099192 & -1.8033825 \\
\hline 10 & $\mathrm{C}$ & $\mathrm{C} 6$ & -.5431990 & .4741667 & -.6966994 \\
\hline 11 & $\mathrm{H}$ & $\mathrm{H} 7$ & -.2114506 & -2.4730371 & -2.1373789 \\
\hline 12 & $\mathrm{H}$ & H8 & -1.3971731 & -.0357959 & -2.6847747 \\
\hline 13 & $\mathrm{H}$ & $\mathrm{H} 10$ & -.8270953 & 1.5000119 & -.5152750 \\
\hline
\end{tabular}


Table s11. Cartesian coordinates $(\AA)$ for 3C neutral.

\begin{tabular}{|c|c|c|c|c|c|}
\hline & & Atom & $\mathrm{X}$ & $\mathrm{Y}$ & $\mathrm{Z}$ \\
\hline 1 & $\mathrm{H}$ & $\mathrm{H} 1$ & 1.1700011 & -.6163542 & -1.7448906 \\
\hline 2 & $\mathrm{C}$ & $\mathrm{C} 1$ & 1.1553642 & -.4124161 & -.6717850 \\
\hline 3 & $\mathrm{~N}$ & $\mathrm{~N} 1$ & 2.2160683 & -.5428053 & .0412550 \\
\hline 4 & $\mathrm{C}$ & $\mathrm{C} 2$ & -.1045970 & .0058891 & -.1070719 \\
\hline 5 & 0 & 01 & 3.2895589 & -.9585232 & -.7612821 \\
\hline 6 & $\mathrm{H}$ & $\mathrm{H} 2$ & 4.0138306 & -1.0204089 & -.1191413 \\
\hline 7 & $\mathrm{O}$ & $\mathrm{O} 2$ & -.1901869 & .2780011 & 1.2310574 \\
\hline 8 & $\mathrm{C}$ & $\mathrm{C} 4$ & -1.4756409 & .6440346 & 1.4813628 \\
\hline 9 & $\mathrm{C}$ & $\mathrm{C} 5$ & -2.2192966 & .6144779 & .3388613 \\
\hline 10 & $\mathrm{C}$ & $\mathrm{C} 6$ & -1.3290757 & .1991936 & -.6973556 \\
\hline 11 & $\mathrm{H}$ & $\mathrm{H} 7$ & -1.6980644 & .8911766 & 2.5084231 \\
\hline 12 & $\mathrm{H}$ & H8 & -3.2684537 & .8571945 & .2452982 \\
\hline 13 & $\mathrm{H}$ & $\mathrm{H} 10$ & -1.5595079 & .0605404 & -1.7447311 \\
\hline
\end{tabular}

Table $\mathbf{5 1 2}$. Cartesian coordinates $(\AA)$ for $3 \mathbf{C}$ radical cation.

\begin{tabular}{|c|c|c|c|c|c|}
\hline & & Atom & $\mathrm{X}$ & $\mathrm{Y}$ & $\mathrm{Z}$ \\
\hline & & & & & \\
\hline 1 & $\mathrm{H}$ & $\mathrm{H} 1$ & 1.1616952 & -.6221869 & -1.7816101 \\
\hline 2 & $\mathrm{C}$ & $\mathrm{C} 1$ & 1.1222476 & -.4128172 & -.7129068 \\
\hline 3 & $\mathrm{~N}$ & $\mathrm{~N} 1$ & 2.1961697 & -.5367322 & .0525913 \\
\hline 4 & $\mathrm{C}$ & $\mathrm{C} 2$ & -.0858806 & -.0042486 & -.1334857 \\
\hline 5 & 0 & 01 & 3.2394220 & -.9317370 & -.6809139 \\
\hline 6 & $\mathrm{H}$ & $\mathrm{H} 2$ & 3.9880828 & -.9985758 & -.0525506 \\
\hline 7 & $\mathrm{O}$ & $\mathrm{O} 2$ & -.1515769 & .2639801 & 1.2116349 \\
\hline 8 & $\mathrm{C}$ & $\mathrm{C} 4$ & -1.4122821 & .6265468 & 1.4736283 \\
\hline 9 & $\mathrm{C}$ & $\mathrm{C} 5$ & -2.2027280 & .6072582 & .3195371 \\
\hline 10 & $\mathrm{C}$ & $\mathrm{C} 6$ & -1.3622219 & .2054278 & -.7100275 \\
\hline 11 & $\mathrm{H}$ & $\mathrm{H} 7$ & -1.6323728 & .8738221 & 2.5041526 \\
\hline 12 & $\mathrm{H}$ & H8 & -3.2531075 & .8582722 & .2654867 \\
\hline 13 & $\mathrm{H}$ & $\mathrm{H} 10$ & -1.6074477 & .0709903 & -1.7555363 \\
\hline
\end{tabular}


Table S13. Cartesian coordinates ( $\AA$ ) for 4A neutral.

\begin{tabular}{rrrrrr} 
& & Atom & \multicolumn{1}{c}{ X } & Y \\
1 & C & C2 & -.0451522 & -.1898157 & .3343493 \\
2 & C & C1 & -.0069153 & -.4413664 & 1.7688831 \\
3 & N & N1 & .7817565 & -.0249539 & 2.6985577 \\
4 & H & H3 & -.7906800 & -1.0987200 & 2.1433392 \\
5 & O & O1 & 1.7937506 & .8572302 & 2.3358533 \\
6 & S & S1 & 1.3035322 & .3892236 & -.6362322 \\
7 & C & C4 & .3646699 & .3057616 & -2.0883353 \\
8 & C & C5 & -.9006370 & -.1598089 & -1.8502631 \\
9 & C & C6 & -1.1312990 & -.4445319 & -.4774327 \\
10 & H & H7 & .8084247 & .5922065 & -3.0325670 \\
11 & H & H8 & -1.6424381 & -.2978433 & -2.6289758 \\
12 & H & H10 & -2.0704213 & -.8234090 & -.0876355 \\
13 & H & H2 & 1.5354091 & 1.3360270 & 1.5204591
\end{tabular}

Table $\mathbf{S 1 4}$. Cartesian coordinates $(\AA)$ for $4 \mathrm{~A}$ radical cation.

$\begin{array}{rllrrr} & & \text { Atom } & & \text { X } & \text { Z } \\ 1 & - \text { C } & \text { C2 } & -.0473853 & -.1580937 & .3267701 \\ 2 & \text { C } & \text { C1 } & -.0403258 & -.3804576 & 1.7157618 \\ 3 & \text { N } & \text { N1 } & .9058098 & -.1381626 & 2.6182159 \\ 4 & \text { H } & \text { H3 } & -.8798096 & -.9292277 & 2.1396674 \\ 5 & \text { O } & \text { O1 } & 1.8445171 & .7630838 & 2.3576504 \\ 6 & \text { S } & \text { S1 } & 1.3050172 & .4449671 & -.6501939 \\ 7 & \text { C } & \text { C4 } & .3602805 & .3075208 & -2.0704554 \\ 8 & \text { C } & \text { C5 } & -.9229953 & -.2021057 & -1.8470159 \\ 9 & \text { C } & \text { C6 } & -1.1504675 & -.4814193 & -.5060981 \\ 10 & \text { H } & \text { H7 } & .7882127 & .5960262 & -3.0246663 \\ 11 & \text { H } & \text { H8 } & -1.6391537 & -.3646441 & -2.6439874 \\ 12 & \text { H } & \text { H10 } & -2.0734989 & -.8910962 & -.1092585 \\ 13 & \text { H } & \text { H2 } & 1.5497987 & 1.4336090 & 1.6936099\end{array}$


Table S15. Cartesian coordinates $(\AA)$ for 4B neutral.

\begin{tabular}{|c|c|c|c|c|c|}
\hline & & Atom & $\mathrm{X}$ & $\mathrm{Y}$ & $\mathrm{Z}$ \\
\hline 1 & $\mathrm{H}$ & $\mathrm{H} 1$ & 1.1677232 & -.6127905 & -2.0003274 \\
\hline 2 & $\mathrm{C}$ & $\mathrm{C} 1$ & 1.1495660 & -.3127714 & -.9497938 \\
\hline 3 & $\mathrm{~N}$ & $\mathrm{~N} 1$ & 2.2474274 & -.1676325 & -.2991544 \\
\hline 4 & $\mathrm{C}$ & $\mathrm{C} 2$ & -.1308927 & -.0817686 & -.3038485 \\
\hline 5 & 0 & 01 & 3.3467851 & -.4434955 & -1.1253985 \\
\hline 6 & $\mathrm{H}$ & $\mathrm{H} 2$ & 4.0969484 & -.3014607 & -.5272071 \\
\hline 7 & $\mathrm{C}$ & $\mathrm{C} 3$ & -.4066837 & .2967808 & .9922190 \\
\hline 8 & $\mathrm{C}$ & $\mathrm{C} 4$ & -1.7977878 & .4225085 & 1.2530416 \\
\hline 9 & $\mathrm{C}$ & $\mathrm{C} 5$ & -2.5745285 & .1409315 & .1592990 \\
\hline 10 & S & $\mathrm{S} 1$ & -1.6127025 & -.2854864 & -1.2147729 \\
\hline 11 & $\mathrm{H}$ & $\mathrm{H} 4$ & .3757073 & .4751734 & 1.7206093 \\
\hline 12 & $\mathrm{H}$ & H6 & -2.2084455 & .7113237 & 2.2146385 \\
\hline 13 & $\mathrm{H}$ & H9 & -3.6531166 & .1586876 & .0806952 \\
\hline
\end{tabular}

Table s16. Cartesian coordinates $(\AA)$ for 4 B radical cation.

\begin{tabular}{|c|c|c|c|c|c|}
\hline & & Atom & $\mathrm{X}$ & $\mathrm{Y}$ & $\mathrm{Z}$ \\
\hline & & & & & \\
\hline 1 & $\mathrm{H}$ & $\mathrm{H} 1$ & 1.1645550 & -.6265997 & -2.0468483 \\
\hline 2 & $\mathrm{C}$ & $\mathrm{C} 1$ & 1.0952869 & -.3263594 & -1.0008835 \\
\hline 3 & $\mathrm{~N}$ & N1 & 2.1952486 & -.1610838 & -.2794386 \\
\hline 4 & $\mathrm{C}$ & $\mathrm{C} 2$ & -.1427861 & -.1019013 & -.3728266 \\
\hline 5 & 0 & 01 & 3.2784347 & -.4118144 & -1.0222401 \\
\hline 6 & $\mathrm{H}$ & $\mathrm{H} 2$ & 4.0423488 & -.2674753 & -.4269579 \\
\hline 7 & $\mathrm{C}$ & C3 & -.3806540 & .2904518 & .9767198 \\
\hline 8 & $\mathrm{C}$ & $\mathrm{C} 4$ & -1.7288738 & .4226088 & 1.2610717 \\
\hline 9 & $\mathrm{C}$ & $\mathrm{C} 5$ & -2.5392280 & .1373966 & .1492298 \\
\hline 10 & S & S1 & -1.6559463 & -.2947009 & -1.2531686 \\
\hline 11 & $\mathrm{H}$ & $\mathrm{H} 4$ & .4285001 & .4591023 & 1.6774045 \\
\hline 12 & $\mathrm{H}$ & H6 & -2.1341042 & .7119128 & 2.2236765 \\
\hline 13 & $\mathrm{H}$ & H9 & -3.6227817 & . 1684626 & .1142614 \\
\hline
\end{tabular}


Table S17. Cartesian coordinates ( $\AA)$ for 4C neutral.

$\begin{array}{rlrrrr} & & \text { Atom } & & \text { X } & \text { Z } \\ 1 & \text { H } & \text { H1 } & 1.2198602 & -.4642707 & -1.7872176 \\ 2 & \text { C } & \text { C1 } & 1.2366555 & -.3876560 & -.6971735 \\ 3 & \text { N } & \text { N1 } & 2.3100025 & -.6300625 & -.0356475 \\ 4 & \text { C } & \text { C2 } & .0101464 & -.0056346 & -.0315442 \\ 5 & \text { O } & \text { O1 } & 3.3579335 & -.9801245 & -.8974069 \\ 6 & \text { H } & \text { H2 } & 4.0915599 & -1.1328537 & -.2813249 \\ 7 & \text { S } & \text { S1 } & -.1119962 & .1513453 & 1.7083641 \\ 8 & \text { C } & \text { C4 } & -1.7835778 & .6001908 & 1.5878051 \\ 9 & \text { C } & \text { C5 } & -2.2194738 & .6256355 & .2904478 \\ 10 & \text { C } & \text { C6 } & -1.1953118 & .2803237 & -.6346967 \\ 11 & \text { H } & \text { H7 } & -2.3471565 & .8182545 & 2.4855501 \\ 12 & \text { H } & \text { H8 } & -3.2331278 & .8822499 & .0029415 \\ 13 & \text { H } & \text { H10 } & -1.3355142 & .2426022 & -1.7100973\end{array}$

Table s18. Cartesian coordinates $(\AA)$ for $4 \mathbf{C}$ radical cation.

$\begin{array}{rlrrrr} & & \text { Atom } & \text { X } & \text { Y } & \text { Z } \\ 1 & \text { H } & \text { H1 } & 1.2114351 & -.4659585 & -1.8215285 \\ 2 & \text { C } & \text { C1 } & 1.1965266 & -.3803490 & -.7343448 \\ 3 & \text { N } & \text { N1 } & 2.2853646 & -.6219417 & -.0188743 \\ 4 & \text { C } & \text { C2 } & .0219701 & -.0114064 & -.0616081 \\ 5 & \text { O } & \text { O1 } & 3.3058252 & -.9596448 & -.8124431 \\ 6 & \text { H } & \text { H2 } & 4.0624353 & -1.1186354 & -.2114398 \\ 7 & \text { S } & \text { S1 } & -.0644837 & .1383921 & 1.7021297 \\ 8 & \text { C } & \text { C4 } & -1.7099527 & .5791900 & 1.5831537 \\ 9 & \text { C } & \text { C5 } & -2.2013069 & .6198710 & .2652621 \\ 10 & \text { C } & \text { C6 } & -1.2282299 & .2873694 & -.6633673 \\ 11 & \text { H } & \text { H7 } & -2.2740420 & .7970737 & 2.4838457 \\ 12 & \text { H } & \text { H8 } & -3.2249526 & .8831115 & .0260051 \\ 13 & \text { H } & \text { H10 } & -1.3805890 & .2529280 & -1.7367906\end{array}$

